\title{
Notch signaling is a critical initiator of roof plate formation as revealed by the use of RNA profiling of the dorsal neural tube
}

\author{
Shai Ofek ${ }^{1 \dagger}$, Sophie Wiszniak ${ }^{2 \dagger}$, Sarah Kagan ${ }^{1}$, Markus Tondl ${ }^{2}$, Quenten Schwarz ${ }^{2^{*}}$ and Chaya Kalcheim ${ }^{1 *}$ (D)
}

\begin{abstract}
Background: The dorsal domain of the neural tube is an excellent model to investigate the generation of complexity during embryonic development. It is a highly dynamic and multifaceted region being first transiently populated by prospective neural crest (NC) cells that sequentially emigrate to generate most of the peripheral nervous system. Subsequently, it becomes the definitive roof plate (RP) of the central nervous system. The RP, in turn, constitutes a patterning center for dorsal interneuron development. The factors underlying establishment of the definitive RP and its segregation from NC and dorsal interneurons are currently unknown.

Results: We performed a transcriptome analysis at trunk levels of quail embryos comparing the dorsal neural tube at premigratory NC and RP stages. This unraveled molecular heterogeneity between NC and RP stages, and within the RP itself. By implementing these genes, we asked whether Notch signaling is involved in RP development. First, we observed that Notch is active at the RP-interneuron interface. Furthermore, gain and loss of Notch function in quail and mouse embryos, respectively, revealed no effect on early NC behavior. Constitutive Notch activation caused a local downregulation of RP markers with a concomitant development of dl1 interneurons, as well as an ectopic upregulation of RP markers in the interneuron domain. Reciprocally, in mice lacking Notch activity, both the $\mathrm{RP}$ and $\mathrm{dl} 1 \mathrm{interneurons}$ failed to form and this was associated with expansion of the $\mathrm{dl} 2$ population.
\end{abstract}

Conclusions: Collectively, our results offer a new resource for defining specific cell types, and provide evidence that Notch is required to establish the definitive RP, and to determine the choice between RP and interneuron fates, but not the segregation of RP from NC.

Keywords: BMP, Quail embryos, delta1, Dorsal interneurons, Fate segregation, Mib1, Mouse embryos, Neural crest, Notch

\footnotetext{
* Correspondence: Quenten.Schwarz@unisa.edu.au; kalcheim@cc.huji.ac.il

tShai Ofek and Sophie Wiszniak contributed equally to this work.

${ }^{2}$ Centre for Cancer Biology, University of South Australia and SA Pathology,

North Terrace, Adelaide, SA 5001, Australia

${ }^{1}$ Department of Medical Neurobiology, Institute of Medical Research

Israel-Canada (IMRIC) and the Edmond and Lily Safra Center for Brain

Sciences (ELSC), Hebrew University of Jerusalem-Hadassah Medical School,

P.O.Box 12272, 9112102 Jerusalem, Israel
}

(c) The Author(s). 2021 Open Access This article is licensed under a Creative Commons Attribution 4.0 International License, which permits use, sharing, adaptation, distribution and reproduction in any medium or format, as long as you give appropriate credit to the original author(s) and the source, provide a link to the Creative Commons licence, and indicate if changes were made. The images or other third party material in this article are included in the article's Creative Commons licence, unless indicated otherwise in a credit line to the material. If material is not included in the article's Creative Commons licence and your intended use is not permitted by statutory regulation or exceeds the permitted use, you will need to obtain permission directly from the copyright holder. To view a copy of this licence, visit http://creativecommons.org/licenses/by/4.0/ The Creative Commons Public Domain Dedication waiver (http://creativecommons.org/publicdomain/zero/1.0/) applies to the data made available in this article, unless otherwise stated in a credit line to the data. 


\section{Summary statement}

A new set of genes involved in Notch-dependent roof plate formation is unraveled by transcriptome analysis.

\section{Background}

Defining the mechanisms that generate a sequence of specialized cell types and further coordinate their functions is critical for understanding organ development. The dorsal neural tube (NT) epithelium is an excellent system to tackle these questions as it is a highly dynamic structure in both time and space [1]. Initially, it is transiently populated by premigratory neural crest (NC) cells. The latter exit the NT to form the peripheral nervous system as well as a rich assortment of additional derivatives that vary along the neuraxis [2-4]. Subsequently, the dorsal NT midline domain becomes the definitive roof plate (RP) of the spinal cord which is flanked ventrally by dorsal interneuron populations $[1,3,5,6]$.

The RP acts as a neural patterning center that secretes growth factors of the bone morphogenetic protein (BMP) and Wnt families, both crucial for dorsal interneuron development [7-14], and for proliferation of ependymal cells at later stages $[15,16]$.

The morphology of the RP differs along the axis. In the hindbrain, it is composed of a large sheet of cells, whereas more caudally, it is a relatively thin, wedgeshaped strip of cells located along the midline of the spinal cord $[17,18]$. Fate mapping analysis revealed that the RP generates the choroid plexus at cranial levels of the axis $[17,18]$. In addition, the RP is transformed into radial glia-like cells $[16,19-21]$, recently found to support growth of spinal cord axons [22]. These radial glial cells eventually generate the stem cell-containing dorsal ependymal zone in the adult spinal cord of humans and rodents $[15,16,23]$.

In spite of its pivotal significance, the origin of the RP in the NT and how it segregates from the earlier NC remained elusive for many years. In fact, the closing dorsal NT was classically termed "RP," yet, essentially, it included the premigratory cohort of NC [1]. To note, several differences exist between these two populations, whereas $\mathrm{NC}$ cells actively proliferate and RP progenitors withdraw from the cell cycle [5, 24]. Moreover, BMP signaling is needed for NC emigration and for initial RP formation, yet the consolidating RP becomes refractory to BMP activity even if it continues synthesizing the protein [5].

To begin understanding the relationship between NC and RP, we previously implemented spatio-temporally controlled lineage analysis in the avian trunk. This revealed first, that the dorsal NT is sequentially transited by distinct cell populations that emigrate to yield NC derivatives $[25,26]$; second, that RP progenitors originate ventral to the premigratory $\mathrm{NC}$; and third, that these $\mathrm{RP}$ precursors relocate ventro-dorsally to reach their final position in the dorsal midline of the NT upon completion of NC exit [25]. Furthermore, tracing the dynamics of the NC marker Foxd3 using a specific reporter revealed that $\mathrm{NC}$ and $\mathrm{RP}$ progenitors are initially part of the Foxd3 lineage yet RP precursors downregulate Foxd3 while relocating into the dorsal midline, thus segregating from the NC [26]. The dorsal NT is, therefore, a dynamic area in which progressive NC emigration takes place until replacement by the definitive RP, resulting in the separation between central and peripheral branches of the nervous system $[1,25]$.

The precedent results provided the basis for understanding the transition between $\mathrm{NC}$ and RP stages. Nevertheless, a serious limitation for further mechanistic understanding of this process was the lack of differential markers expressed in either population. Whereas several $\mathrm{NC}$-specific genes are known (e.g., Foxd3, Sox9, Snail2), many of them (e.g., Lmx1a, Lmx1b, Msx, BMP4, Wnt1) continue to be expressed in RP, and only few are known to be expressed uniquely in the latter (e.g., cHes1/ Hairy1, R-spo1) [5, 27]. For elucidating RP formation, availability of such unique genes is of utmost significance to serve both for identification purposes as well as for functional studies.

In addition to its separation from the $\mathrm{NC}$ lineage, a boundary separates the ventral domain of the nascent $\mathrm{RP}$ and prospective dorsal interneurons along the spinal cord. Although the RP secretes BMP and Wnt proteins responsible for specification and differentiation of selected interneuron cell types (see above), the factors that determine the establishment of the RP and/or the formation of the boundary between RP and interneurons remain unknown. At cranial levels, maintenance of the boundary between RP and the rhombic lip neuroepithelium was shown to be mediated by Delta (Dll)-Notch signaling but its initial formation was not investigated, neither at cranial levels, nor in the trunk [18]. Notch and its ligands are ubiquitously used for coordination of differentiation between neighboring cells in processes such as boundary formation, lateral inhibition, and cell fate determination [28-30].

In the present study, we performed a transcriptome analysis at trunk levels of quail embryos comparing the dorsal NT domain at premigratory NC and RP stages. This analysis yielded genes that were differentially and specifically expressed in either NC or RP. Furthermore, we unraveled a molecular heterogeneity within the trunk-level RP, with transcripts expressed mainly in its lateral or medial domains, respectively. Collectively, our results provide a new resource for investigating a fundamental fate transition during neural development. By implementing the newly described genes in both quail and mouse embryos, we further examined the hypothesis that Notch signaling is involved in de novo RP 
development in the trunk. First, we observed that Notch is active in the dorsal NT except for the dorsal midline region and a similar dorsal limit of mRNA expression was observed for Dll1, Serrate/Jagged, and Lfng, altogether suggesting that Dll-Notch signaling operates at the boundary between the prospective RP and dorsal interneurons. Gain of Notch function in quails revealed a cell-autonomous inhibition of RP markers and their replacement by dI1 interneurons. Additionally, an ectopic induction of the expression of RP traits was apparent ventral to the endogenous RP and this was associated with reduced expression of BarHL1, a dI1 interneuron marker. Reciprocally, loss of Notch function was performed in mice by tissue specific knockout of the E3 ubiquitin ligase mindbomb1 (Mib1) to remove the function of all Notch ligands in the dorsal NT and its derivatives. In Wnt1-Cre; MibI $1^{f l f l}$ embryos, no RP or dI1 interneurons developed. Concomitantly, dI2-dorsal interneurons expanded dorsal-ward. In contrast, both gain and loss of Notch function revealed no effect on emigration/migration of NC cells. Together, we provide evidence that Notch signaling is both sufficient and necessary for the choice between RP and interneuron fates without affecting early stages of NC ontogeny.

\section{Results}

\section{Transcriptional profiling of the dorsal NT at early} premigratory NC and RP stages

To conduct a genome-wide transcriptome analysis of dorsal NT development during the transition from NC to RP stages, we electroporated a GFP-encoding plasmid into the dorsal domain of quail NTs at either stage (18ss for $\mathrm{NC}$ and 43ss for RP, respectively, Additional file 1: Fig. S1A,B,E,F). Six to $8 \mathrm{~h}$ later, NTs were isolated and dissociated into single cells, and fluorescent cells were FACSsorted. No GFP+ cells were apparent in control, nonelectroporated samples (Additional file 1: Fig. S1C, G). In contrast, and as expected from focal transfections, the percentage of live, GFP + cells was $0.32 \%$ and $0.28 \%$ of the total input for NC and RP, respectively. In both control and electroporated cases, very low proportions of propidium iodide (PI)+ dead cells were found; these were excluded by gating and only 1000-6000 GFP+/PI- cells/ sample were collected (Additional file 1: Fig. S1D, H).

Next, harvested cells were processed for RNAsequencing (RNA-seq) in triplicates for each stage (Fig. 1a-c). Principal component analysis (PCA) showed that NC and RP samples cluster into two distinct groups (Fig. 1a). Differential gene expression analysis revealed significant upregulation of 1243 and downregulation of 1068 genes in RP when compared to NC, respectively, providing a rich data source for further functional analysis (Fig. 1b, c and Additional file 2: Table S1 and Additional file 3: Table S2).
Further examination by ingenuity pathway analysis (IPA), revealed functional categories containing genes with significant expression changes. Those which were enriched in RP compared to NC (Fig. 1d) included "development of the body trunk," "development of central nervous system," and "development of the spinal cord (SC), reflecting that the RP is a part of the central nervous system. Furthermore, among the RP enriched categories was "epithelial tissue development," consistent with our previous findings showing that during the transition between NC and RP, the latter regains epithelial characteristics [5]. Enrichment of additional categories such as "guidance of axons" and "midline defects" are also consistent with the established role of the RP as a guidance center for adjacent interneuron projections and that RP abnormalities are a source of NT closure defects, such as spina bifida [31, 32]. The presence of "differentiation of neurons" in this analysis may possibly arise because the RP secretes necessary factors for interneuron differentiation $[12,33]$ and/or due to the inclusion of interneuron progenitors located adjacent to the $\mathrm{RP}$ in the sorted cell suspensions.

Ingenuity pathway analysis uncovered cell cycle regulation as the major functional group downregulated in RP compared to NC (Fig. 1e). This is consistent with the marked change in proliferative properties, as revealed by BrdU incorporation, between mitotically active premigratory $\mathrm{NC}$ compared to the definitive RP, that progressively turns into a post-mitotic structure $(45.9 \pm 4 \%$ vs. $4.8 \pm 0.5 \%$, respectively, Fig. $1 \mathrm{f}-\mathrm{h}$ ) (see also [5]). Along this line, $M y c N$ mRNA, a marker of proliferative cells [34], was present in the dorsal NT at the NC stage, yet was downregulated in RP (Fig. 1i, j). Additional cell cycle associated genes were reduced in RP by at least threefold, such as Cdk2-Ap2, olfactomedin4, S100A11, Dlg1, cyclin D3, and Rgcc (Supplementary Table S1), whereas cell cycle inhibitors were upregulated (Cdkn2c, Cdkn1c, Dach1 and 2, Btg11, Ndrg1 (Additional file 3: Table S2).

Taken together, enrichment of the above categories substantiate the validity of our molecular analysis in exposing well-known differential traits of premigratory $\mathrm{NC}$ versus RP populations.

\section{The expression patterns of differential genes NC versus $R P$ markers}

To further validate our RNAseq results, we examined by in situ hybridization (ISH) the localization of genes known to be differentially expressed in either NC or RP, as well as that of transcripts never tested before in this context. We present a subset of them alongside graphical representations of their normalized expression based on triplicate samples at either stage. Consistent with our previous results $[25,26,35]$, the NC markers FoxD3 and Snail2 were downregulated at the RP stage 


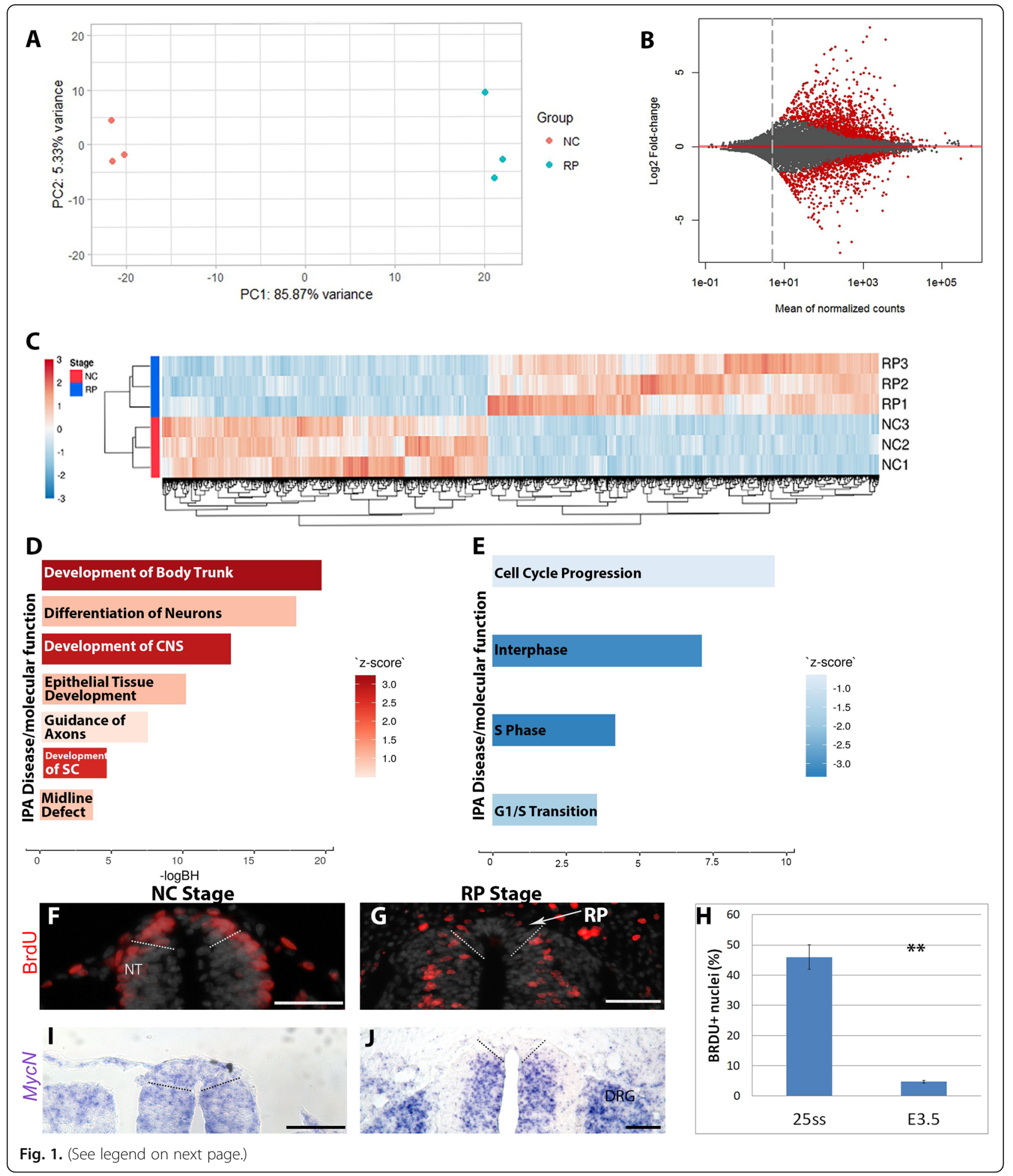


(See figure on previous page.)

Fig. 1. Transcriptome analysis of small groups of premigratory NC and RP cells. a Principal component analysis shows a distinct segregation of transcripts for the purified populations of NC and RP cells along 2 principal components axes (PC1, PC2). $\mathbf{b}$ MA plot comparing RP over NC genes. The plot visualizes the differences between measurements taken in two samples, by transforming the data onto $\mathrm{M}$ (log ratio) and A (mean average) scales. Genes upregulated in RP when compared to NC are above the red line and downregulated genes are below. $\mathbf{c}$ Heat map of 2171 differentially expressed genes (padj< 0.05) between NC and RP. Red and blue colors represent all significantly upregulated and downregulated genes, respectively. Normalized expression is shown after scaling the values for each gene. Genes and samples are ordered by

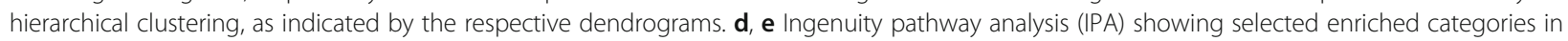
RP with reference to NC (red) or downregulated in RP/NC (blue), that include molecular functions and diseases ( $p$ value $<0.05)$. Categories with IPA Z-scores greater than 2 or smaller than -2 can be considered to be significantly predicted to be up- (d, red) or down- (e, blue) regulated, respectively. $\mathbf{f}-\mathbf{h}$ Bromodeoxyuridine (BrdU) incorporation following a 1-h pulse at NC (E2, f) or RP (E3.5, g) stages. Note the presence of the BrdU+ nuclei (red) in NC domain but not in RP (delimited by dashed lines). Quantification of Brdu-positive cells (H) shows significantly reduced number of proliferating cells in the RP $\left({ }^{*} p<0.001, N=5 /\right.$ stage). $\mathbf{i}, \mathbf{j}$ ISH for MycN shows downregulation in RP (delimited by dashed lines). Abbreviations, DRG, dorsal root ganglion, NT, neural tube. Bar $=50 \mu \mathrm{m}$

(Additional file 4: Fig. S2A-F). Notably, so were the Wnt inhibitors Dact2, Sfrp2, and Dkk1 (Additional file 4: Fig. S2G-O). In all cases, there was a positive correlation between the transcriptome data and the ISH patterns. Additional transcripts significantly reduced in RP when compared to NC were Ltk, Mesp1, Runx1, Calponin2, ETS1, ETS2, Glipr2, Ptn, Ripply1, LMO4, Mylk, SALL4, syndecan4, Cdx2, Cdx4-like, Spry2, Nrp2, Tfap2C, Tspan1, Sox18, Mef2C, and Gata5 (Additional file 2: Table S1). Cell adhesion molecules also changed between the stages, with Cad1, Cad6, Cad11, Cad20, Pcdh1, Pcdh19, and B-cadherin-like, being downregulated in RP. Among upregulated cell adhesion genes were Pcdh8, Pcdh9, Pcdh11, Pcdh18, Cad4, Cad8, Cad22, and SDK2 (Supplementary Table S2). However, N-Cad (Cdh2) mRNA levels did not change, consistent with previous results showing that the dynamics of N-cad function in the dorsal NT is regulated by post-transcriptional processing [36].

Importantly, we found a selection of genes expressed in RP but not premigratory NC (Fig. 2). These included the RPspecific Spondin Rspo1 [27], and HES4, the quail ortholog of chick Hairy1 that exhibits $94 \%$ identity at the protein level. In addition, the BMP member Gdf7, and the BMP antagonists $B A M B I$ and Gremlin, the retinoic acid-associated genes Raldh2 and CRABP1, and the chemorepellents of commissural axons Draxin and Slit1 are also included (Fig. 2).

In the category of genes enriched in RP compared to $\mathrm{NC}$, there were additional transcripts that exhibited a wider expression pattern that included, but was not restricted to the RP. Examples were Norrin (NDP), LRP8, Znf536, and Zic4 (Additional file 5: Fig. S3). In all cases, there was also a good correlation between the differential expression patterns and transcriptome results. Together, our RNAseq provided us with a set of spatiotemporal-specific genes appropriate for cell type identification and for functional studies.

\section{Expression of "non-RP" markers is complementary to the RP-specific genes}

While attempting to validate by ISH the localization of differential transcripts, a subset of genes showing a significant upregulation in the RP (Additional file 6: Fig. S4, right panels) yielded a surprising localization pattern. Dach1, LFNG, CDKN2C, BCL11B, Wnt4, OLFM1, and INSM1 mRNAs were all expressed in different cell subsets along the NT, except for the RP itself which remained negative (Additional file 6: Fig. S4). This observation suggests that results of RNAseq cannot be taken as the sole criterion for marker expression in a given cell type, and further spatial confirmation by ISH is warranted.

We predicted that the precedent genes could prove to be useful in defining the extent of the nascent RP, if delimiting the ventral boundary of this structure. To this end, we performed ISH of adjacent sections at the RP stage, combining the Notch-related gene LNFG with a set of RP-specific transcripts. In all cases examined, the dorsal limit of $L F N G$ expression corresponded to the ventral limit of localization of RSpo1, HES4, BAMBI, Draxin, Raldh2, Gdf7, and Crabp1, respectively (Additional file 7: Fig. S5). Reciprocally, the RP-specific RSpo1 gene was hybridized in combination with various "nonRP" genes such as BCL11B, Dach1, OLFM1, INSM1, and Wnt4, exhibiting a comparable complementary pattern (Additional file 8: Fig. S6, A-O). Hence, the expression of RP and "non-RP" genes enabled us to define the precise size of the RP territory. Measurement of the number of Hoechst-positive nuclei within the RP territory delimited by $L F N G$, revealed that the flank-level RP at E3.5 of quail development consists of $61 \pm 1.92$ cells per $10 \mu \mathrm{m}$ section ( $N=4$, Additional file 8: Fig. S6P-R).

\section{Molecular heterogeneity within the $R P$}

Careful inspection of the patterns of expression of RP genes, revealed that Gremlin1, BAMBI, Raldh2, HES4, and Draxin were homogeneously distributed throughout the RP (Fig. 2 and Additional file 7: Fig. S5). In contrast, the downstream Wnt target Axin2, the Wnt-associated ubiquitin ligase RNF43, and the mRNA encoding the RSpo1 receptor Lgr4 were confined to the medial domain of the RP (Additional file 9: Fig. S7). 

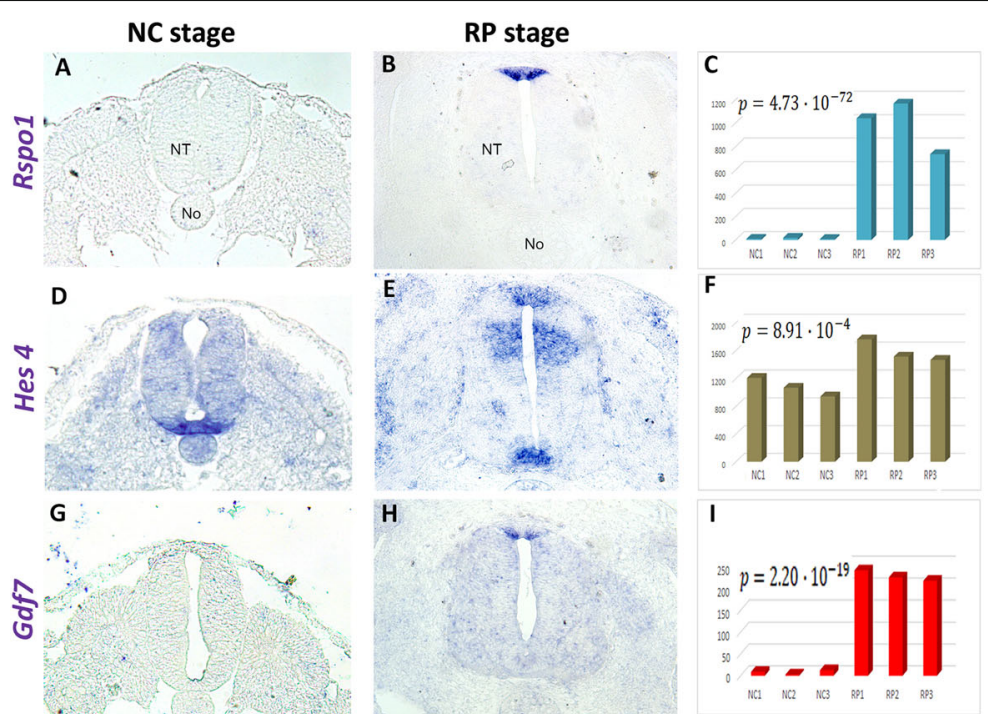

F
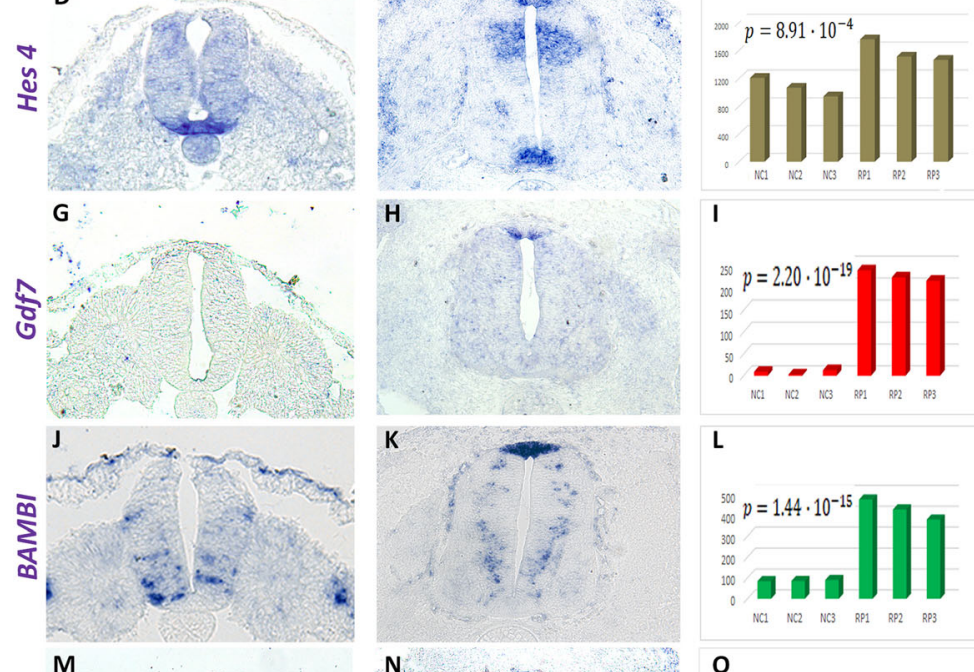

O
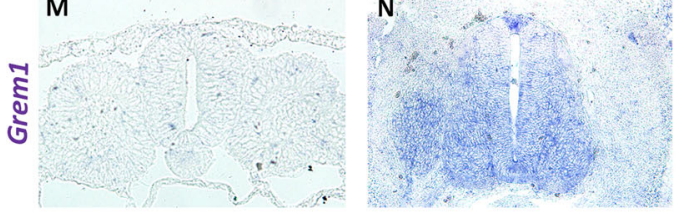

60 $p=4.87 \cdot 10^{-3}$
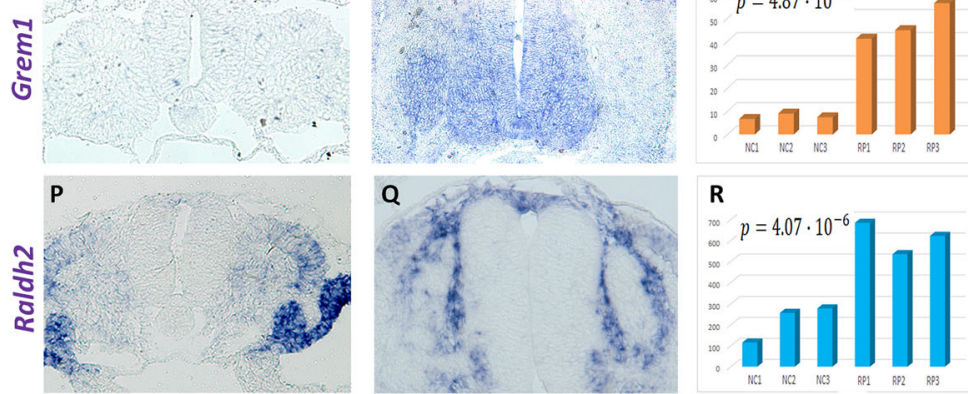

$\mathbf{R}$

${ }_{500}^{200} p=4.07 \cdot 10^{-6}$
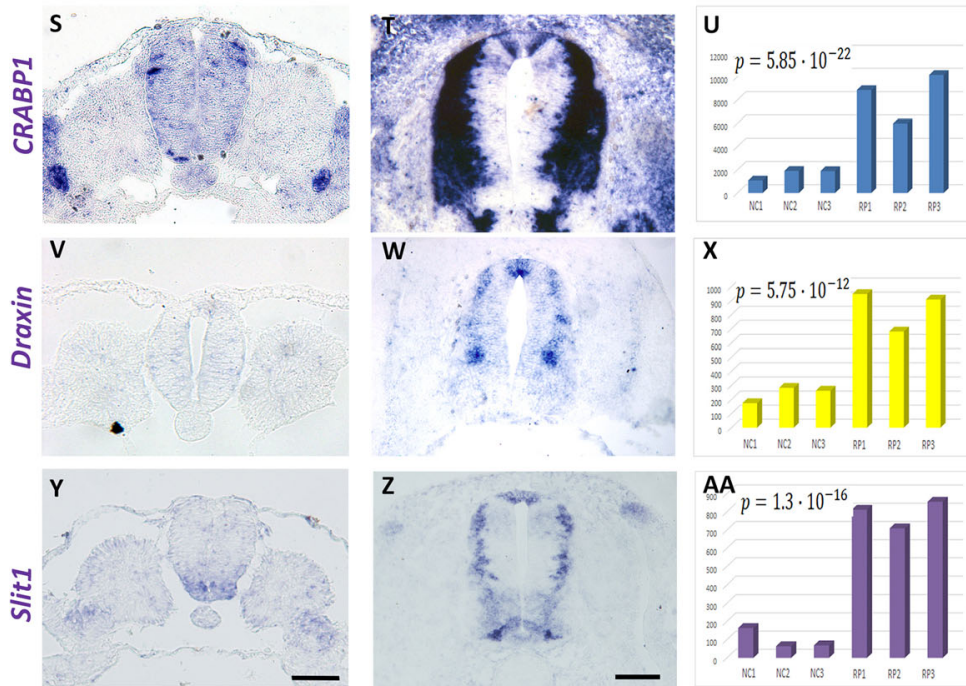

w

$\mathrm{X}$
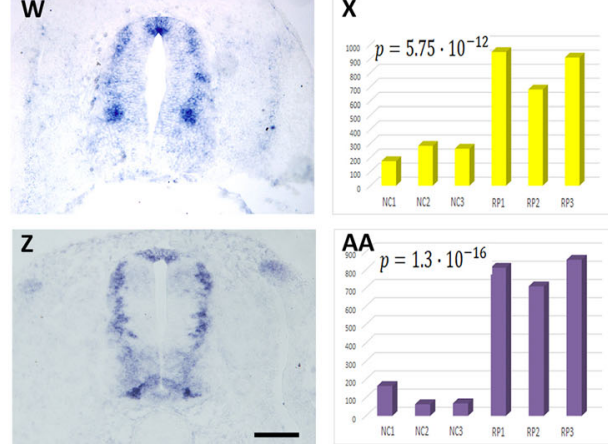

Fig. 2. (See legend on next page.) 
(See figure on previous page.)

Fig. 2. ISH for selected genes upregulated in $R P$ compared to $N C$. $\mathbf{a}, \mathbf{d}, \mathbf{g}, \mathbf{j}, \mathbf{m}, \mathbf{p}, \mathbf{s}, \mathbf{v}, \mathbf{y} I S H$ at $N C$ and $R P(\mathbf{b}, \mathbf{e}, \mathbf{h}, \mathbf{k}, \mathbf{n}, \mathbf{q}, \mathbf{t}, \mathbf{w}, \mathbf{z})$ stages, respectively. Note expression in the dorsal tube at RP but not NC stage. $\mathbf{c}, \mathbf{f}, \mathbf{i}, \mathbf{I}, \mathbf{o}, \mathbf{r}, \mathbf{u}, \mathbf{x}$, aa Quantification of gene expression levels stemming from the transcriptome analysis. Note positive correlation between ISH and transcriptome results. In each chart, the $\mathbf{b}-\mathbf{h}$ adjusted $p$ value is indicated. Abbreviations, NT, neural tube, No, notochord. Bar $=50 \mu \mathrm{m}$

A reciprocal pattern was observed for RSpo1 that was primarily transcribed in two peripheral stripes of the RP delimiting the central domains of expression of Axin2, RNF43, and Lgr4 (Additional file 9: Fig. S7) and abutting the ventral limits of the RP (Additional file 7: Fig. S5A-C and Additional file 8: Fig. S6). A similar pattern was observed for Gdf7 [(Additional file 7: Fig. S5P-R, Fig. 2, see also [18]] and CRABP1 (Additional file 7: Fig. S5S-U, and Fig. 2). Hence, similar to the RP at the hindbrain level [17], the flank-level RP also comprises at least two distinct domains, a medial and two flanking lateral regions (Additional file 9: Fig. S7J).

\section{The role of Notch signaling in RP development}

Notch activity was found to be involved in maintenance of the hindbrain RP [18]. By implementing a subset of the newly identified genes described above, we investigated whether this signaling pathway plays a role in de novo RP formation at spinal cord levels.

\section{Expression and activity of Notch ligands and receptors in the dorsal NT}

Expression of Dll1, Jagged1, and Jagged 2 was evident in various domains of the quail NT at E4, except for the RP; thus, localization of ligand mRNAs was complementary to Rspo1 (Additional file 10: Fig. S8). Likewise, LFNG, that facilitates Notch signaling, exhibited a similar non-RP pattern (Additional file 9: Fig. S7). Notch1 was widespread throughout the ventricular layer of the NT, exhibiting an apical localization in RP, and Notch2 was transcribed in RP (Additional file 10: Fig. S8).

To examine the activity of Notch, we used an antibody specific to its active intracellular domain (ICD) that recognizes the mouse but not the quail protein. Whereas immunolabeling was almost undetectable during the NC stage at E9.75 (Fig. 3a, b), expression of N1ICD was evident at both E10.5 and E11.5 throughout the NT except for the dorsal-most domain corresponding to the nascent and definitive RP, respectively (Fig. 3e, f, i, j). To inactivate Notch signaling in the dorsal NT, we conditionally removed the ubiquitin ligase Mindbomb1 (Mib1) that is required for the functional internalization of all Notch ligands, by intercrossing Mibr ${ }^{f l f l}$ and Wnt1Cre mice [37]. As the Wnt1 promoter drives expression of Cre in the dorsal third of the NT prior to the NC stage, this cross abolishes Notch activity prior to and during RP formation. Indeed, N1ICD immunostaining was specifically absent in the dorsal NT of Wnt1-Cre;
$M i b 1^{f l / f l}$, while still present in more ventral domains (Fig. 3c, d, g, h, k, l), consistent with N1ICD specifically representing Notch activity.

Expression of Dll1 protein exhibited a similar pattern in the wildtype embryos to that observed in quails, with no expression in the dorsal NT and later RP (Fig. 3m, n, q, r, u, v). As expected, Wnt1-Cre; Mibf fl/fl embryos revealed enhanced Dll1 signal, consistent with the role of Mib1 in targeting Notch ligands for ubiquitination and their accumulation at the cell membrane as inactive ligands in its absence [38]. Most importantly, by E10.5, Dll1 expression shifted dorsally with significantly fewer Dll1-negative cells in the dorsal midline region corresponding to the RP, and Dll1 expression reached the dorsal midline by E11.5 with almost no Dll1-negative cells remaining in Wnt1-Cre; Mib1 ${ }^{f l / f l}$ embryos (Fig. 3o, $\mathrm{p}, \mathrm{s}, \mathrm{t}, \mathrm{w}-\mathrm{z}$ ).

Next, we assessed the dorsal boundary of expression of Dll1 relative to N1ICD. As shown in Fig. 3aa, both markers display a similar dorsal border of expression. To further explore whether the dorsal domain of Notch activity overlaps with the RP, N1ICD and Gdf7 ISH were performed in adjacent sections. The domains of Gdf7 expression and Notch activity were juxtaposed with no apparent overlap (Fig. 3bb, cc, dd), consistent with the complementary expression of Notch ligands and RP markers in the quail (Additional file 10: Fig. S8). Taken together, this indicates that the ventral border of the prospective RP, where both ligands and receptors meet, is an area of Notch activity. Furthermore, the dorsal expansion of Dll1 expression in Wnt1-Cre; Mib1 ${ }^{f l / f l} \mathrm{em}$ bryos suggests a requirement for Notch signaling in border formation and RP development.

\section{Gain of Notch function in quail NTs regulates the choice between RP and dI1 interneuron identities without affecting early NC development}

To begin testing the possible involvement of Notch signaling in RP development, we first expressed a constitutively active form of Notch2 (aN2) in embryos aged 25ss, prior to RP formation, followed by fixation at E4 (RP stage). Electroporation of aN2 prevented, in a cellautonomous manner, the upregulation of all the RP markers examined, including Rspo1, BAMBI, Raldh2, and Draxin (Fig. 4, arrows). In contrast, control GFP had no effect on the expression of either gene (Fig. 4, arrowheads). Likewise, electroporation of active Notch1 in RP similarly reduced expression of BAMBI, Raldh2, 


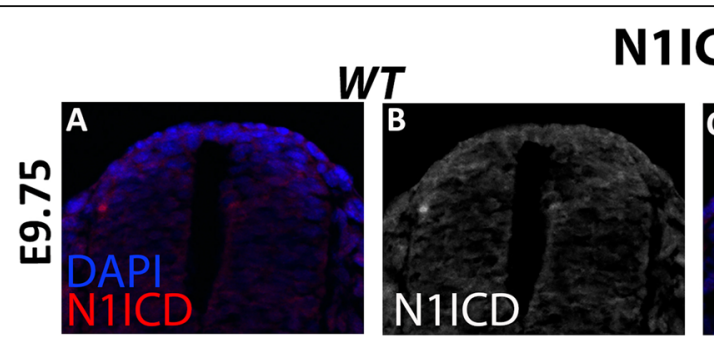

\section{ICD}

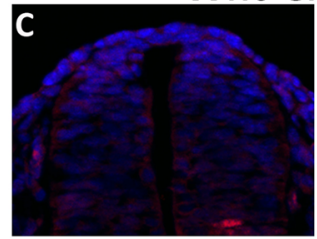

Wnt-Cre;Mib1 $1^{f l / f l}$
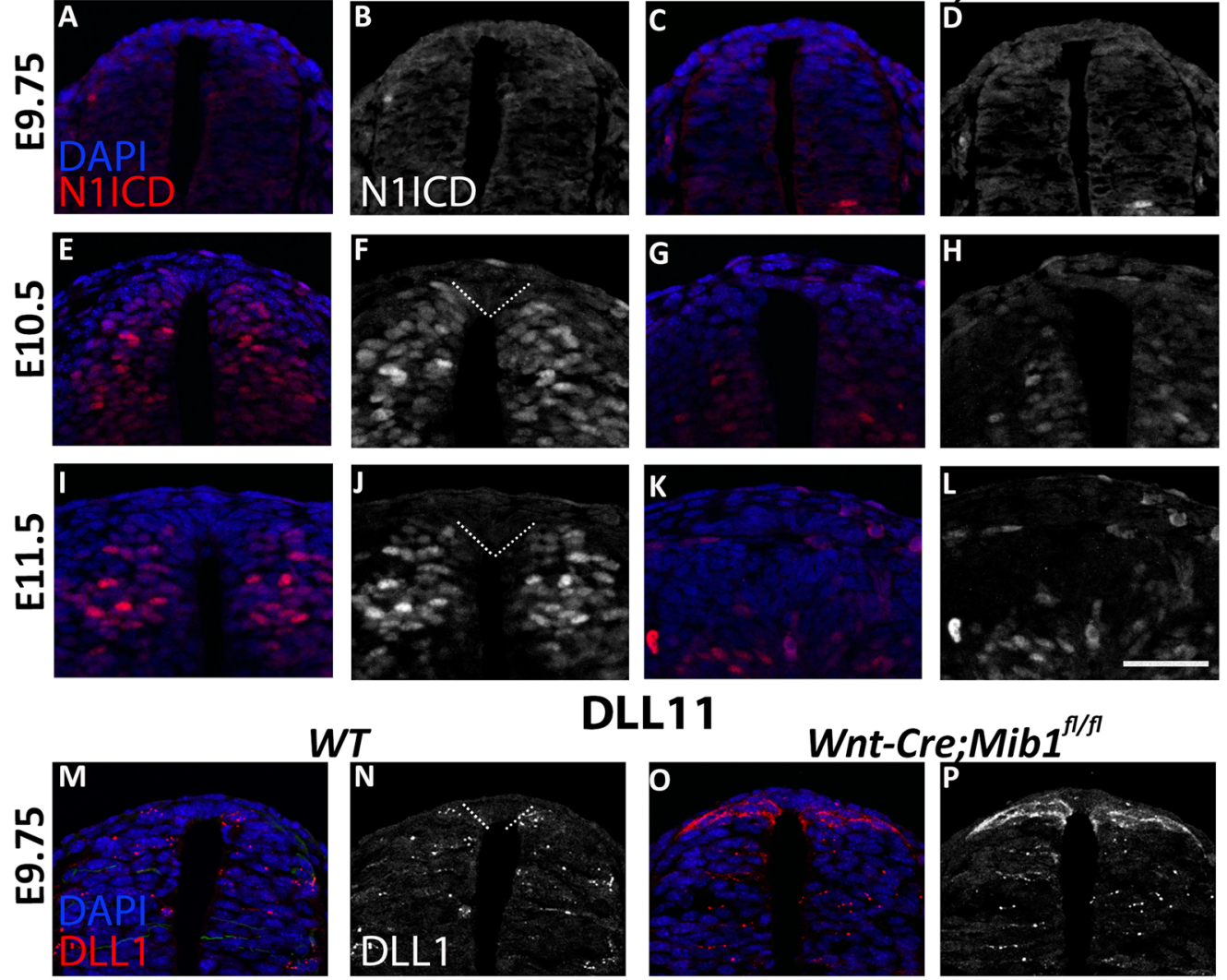

DLL11

WT
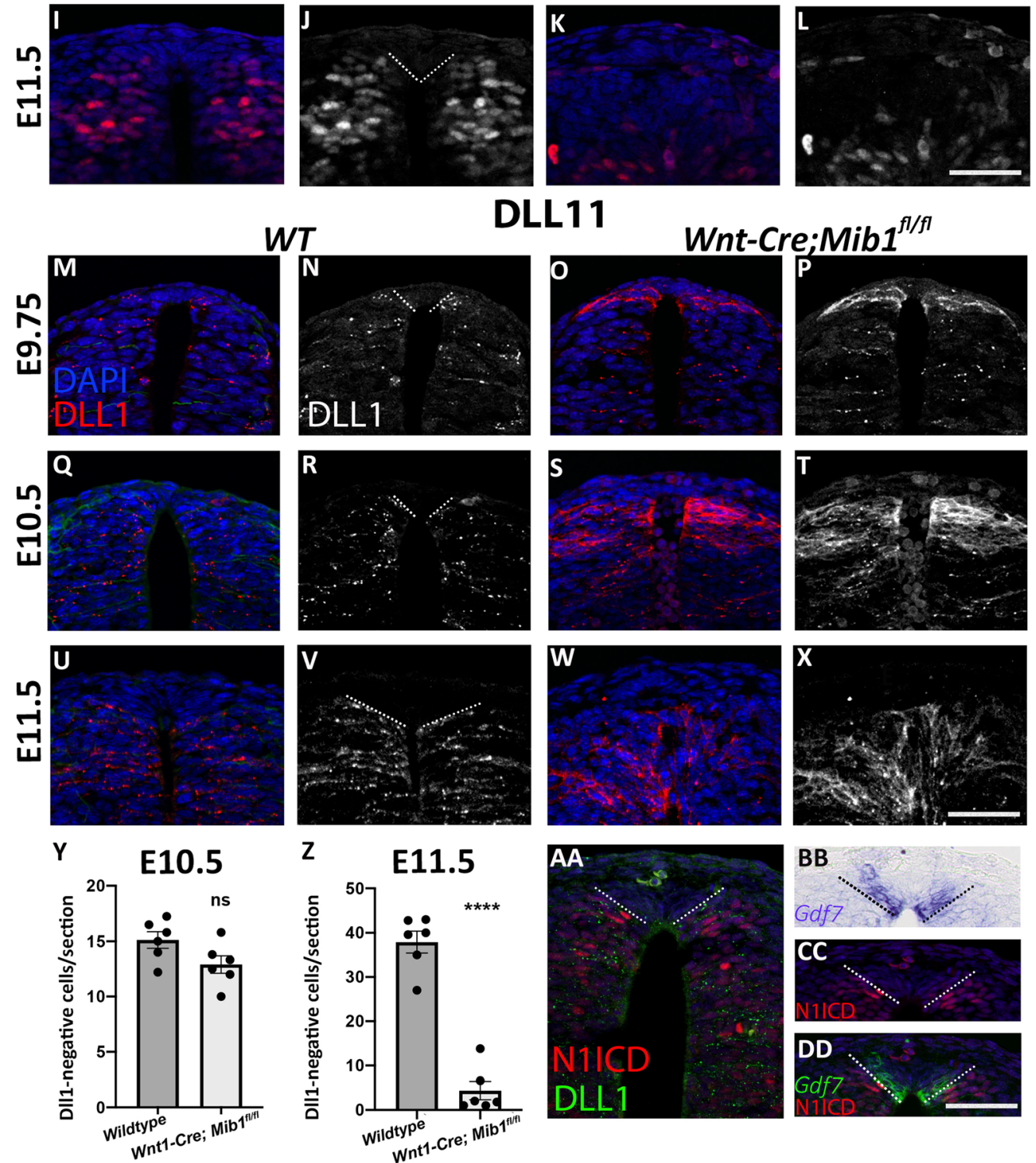

BB

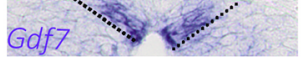

CC

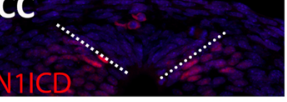

DD

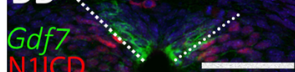

Fig. 3. (See legend on next page.) 
(See figure on previous page.)

Fig. 3. Activation of Notch signaling at the ventral roof plate border. a-I Transverse sections through the dorsal neural tube at the level of the forelimb in wildtype (WT) and Wnt1-Cre; Mib fl/fl $^{f}$ mouse embryos, immunostained for N1ICD (white or red) and DAPI (blue). N1ICD staining is shown in combination with DAPI (a, $\mathbf{c}, \mathbf{e}, \mathbf{g}, \mathbf{i}, \mathbf{k})$ or as a single color image for clarity $(\mathbf{b}, \mathbf{d}, \mathbf{f}, \mathbf{h}, \mathbf{j}, \mathbf{I})$. Low levels of N1ICD are evident in the neural tube at E9.75 (a-d); however, at E10.5 (e-h) and E11.5 (i-I), N1ICD is broadly expressed throughout the dorsal neural tube in wildtype embryos, whereas this is diminished in Wnt1-Cre; Mib $1^{f l / f l}$ embryos. $\mathbf{m}-\mathbf{x}$ Transverse sections through the dorsal neural tube at the level of the forelimb in wildtype (WT) and Wnt1-Cre; Mib $1^{f l / f l}$ mouse embryos, immunostained for DLL1 (red or white) and DAPI (blue). DLL1 staining is shown in combination with DAPI $(\mathbf{m}, \mathbf{o}, \mathbf{q}, \mathbf{s}, \mathbf{u}, \mathbf{w})$ or as a single color image for clarity $(\mathbf{n}, \mathbf{p}, \mathbf{r}, \mathbf{t}, \mathbf{v}, \mathbf{x})$. At all ages examined, in wildtypes, DLL1 is expressed throughout the neural tube except for its dorsal-most region corresponding to the RP (boundary demonstrated by dashed lines in $\mathbf{n}, \mathbf{r}, \mathbf{v}$ ). In Wnt1-Cre; Mib $1^{\text {fl/fl }}$ embryos, DLL1 protein accumulates at the cell membrane due to lack of Mib1-induced endocytosis, and by E1 1.5 there are no DLL1-negative cells present in the dorsal-most neural tube. $\mathbf{y}, \mathbf{z}$ Quantification of DLL1-negative cells in the dorsal-most neural tube at E10.5 and E11.5. At E10.5, there is no difference between genotypes, whereas at E11.5 there is a significant reduction in the number of DLL1-negative cells, and hence of the presumptive RP, in Wnt1-Cre; Mib $1^{\text {fl/fl }}$ embryos. N=6; ns, not significant; ${ }^{* * * *} p<0.0001$. aa Double immunostaining for DII1 (green) and N1ICD (red) at E11.5; note that the dorsal limit of expression of both markers coincides (dotted lines). bb, cc, dd Gdf7 ISH (blue or green) and N1ICD immunostaining (red) of adjacent sections showing a side-by-side expression of both markers with no apparent overlap (dotted lines). Bar $=50 \mu \mathrm{m}$

and Draxin (Additional file 11: Fig. S9), suggesting that both Notch1 and Notch2 have similar functions in this context.

Interestingly, when electroporation of aN2 attained areas adjacent to the RP (Fig. 5, arrows), an ectopic upregulation of Rspo1, BAMBI, Raldh2, and Draxin was often observed more ventrally within the domain populated by dorsal interneurons (Fig. 5, arrowheads). This was not observed upon transfection of control GFP (Fig. 4).

These results suggest that ectopic Notch activation in RP suppresses development of RP-specific traits while inducing them in the adjacent interneuron domain.

To examine the possibility that interneurons developed at the expense of RP cells, we electroporated control GFP or aN2, and sections were subjected to ISH for $B A M B I$ mRNA together with immunolabeling for the dI1 interneuron marker BarHL1. Electroporation of control GFP had no effect on either $B A M B I$ expression in $\mathrm{RP}$ or on the distribution of BarHL1+ interneurons immediately ventral to the RP (Fig. 6a-d). In contrast, many dorsal cells missexpressing aN2 lacked $B A M B I$ mRNA and expressed instead the dI1 interneuron marker; as expected, these differentiated neurons localized to the basal domain of the dorsal NT (Fig. 6e-h, arrowheads). Quantification of BarHL1+ dI1 interneurons within the $B A M B I+$ domain confirmed their ectopic development at the expense of RP (Fig. 6i).

Next, we examined whether gain of Notch activity outside the RP would affect normal dI1 interneuron development. Control GFP did not alter the expression of BarHL1-positive interneurons, whereas aN2-missexpressing cells lacked BarHL1 immunoreactivity (Fig. 6j-0, arrowheads), suggesting a failure of dI1 formation upon excess Notch.

At the time of electroporation (22-25ss), NC cells are already specified in the dorsal NT at the trunk level [39]. To further examine a possible effect of aN2 on $\mathrm{NC} \mathrm{mi-}$ gration, electroporated embryos were fixed a day later. No significant effect was monitored in the number of
GFP-positive NC cells in control vs. aN2-treated embryos (Fig. 6p-t). Altogether, misexpression of active Notch affects the choice between adopting a RP fate or a dI1 interneuron identity, yet has no significant effect on emigration or migration of specified NC progenitors (Figs. 4, 5, and 6).

\section{Failure of RP formation in mutant mice lacking Notch activity in the dorsal NT}

Next, by implementing various RP-specific markers uncovered in the transcriptome analysis of quail embryos, we asked whether Notch signaling is necessary for RP formation in the mouse. Similar to avians, wildtype mouse embryos expressed Rspo1, GDF7, Raldh2, Slit1, $M s x 1$, and Lmx1a in the trunk RP at E11.5 ((Fig. 7a, e, g, $\mathrm{i}, \mathrm{k}, \mathrm{m}$, see also [40]). At variance with the quail, $B A M B I$ was expressed at cranial levels of the mouse axis and was weak to undetectable in the trunk (Fig. $7 \mathrm{c}$, and not shown). Similar to avian embryos, Rspo1, Bambi, and Gdf7 were expressed in the lateral part of the RP, suggesting that this structure is heterogeneous in both species. In striking contrast, no expression of the above transcripts was apparent at E11.5 in Wnt1-Cre; Mibf fl/fl mutants (Fig. 7b, d, f, h, j, l, n). Furthermore, co-labeling of adjacent sections with both Lmxla and Dll1, confirmed the complementary expression of both markers in wildtype embryos, the downregulation of Lmxla in Wnt1-Cre; Mib1 ${ }^{f l f l}$ embryos and the corresponding dorsalization of Dll1 (Fig. 7m, n). Next, we asked whether the loss of the RP genes was due to a failure in "de novo" $\mathrm{RP}$ formation as opposed to its maintenance. To this end, control and mutant embryos were in situ hybridized at E10.5 corresponding to the onset of RP formation, with RP-specific genes not transcribed previously at the NC stage. Expression of Rspo1, BAMBI, GDF7, Raldh2, and Slit1 was undetectable in Wnt1-Cre; Mib1 $1^{\text {fl/fl }} \mathrm{mu}$ tants when compared to wildtype embryos (Fig. 7o-x). Together, our data demonstrate that Notch activity in the dorsal NT is necessary for initiation of RP formation. 

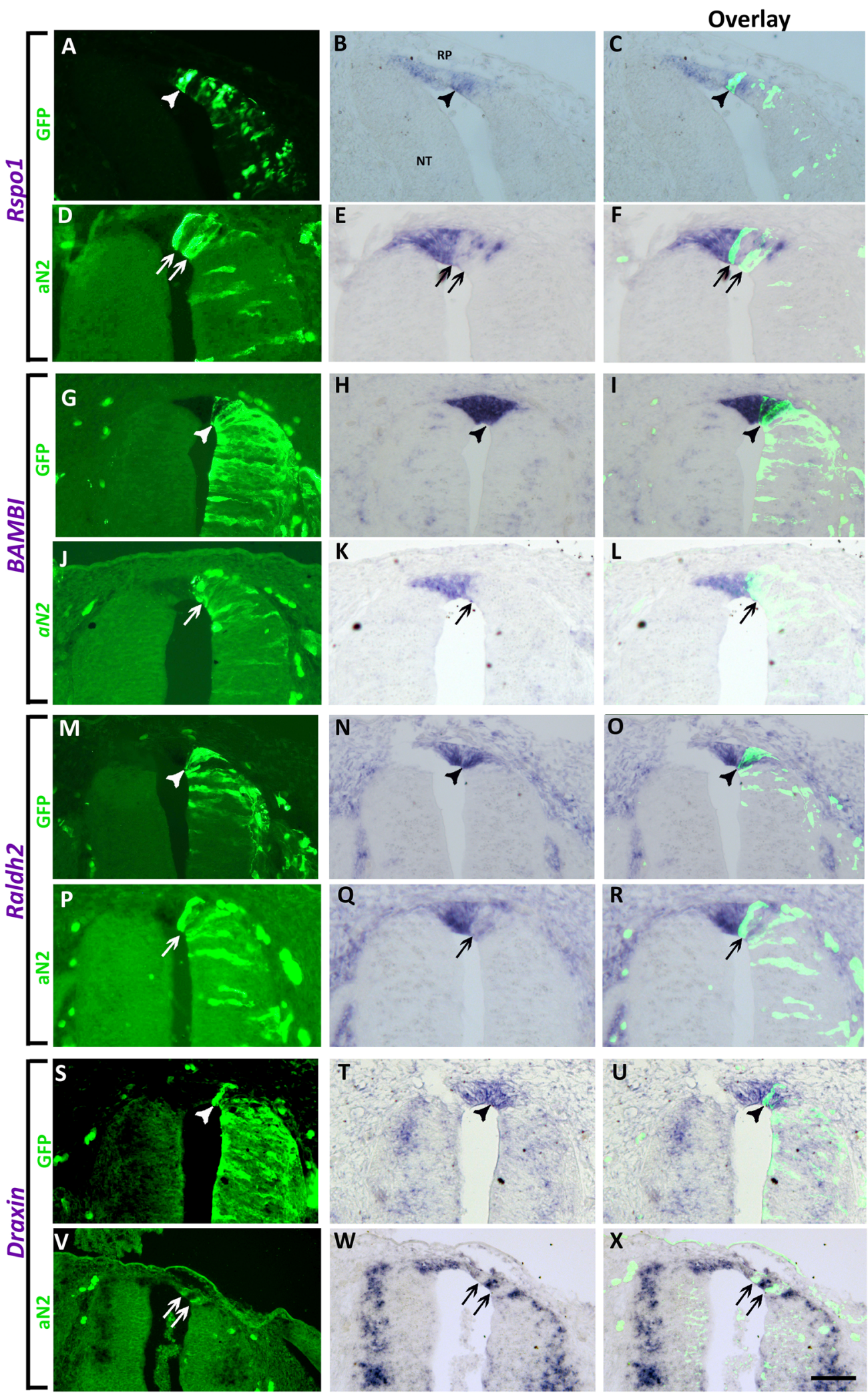

Fig. 4. (See legend on next page.) 
(See figure on previous page.)

Fig. 4. Missexpression of active Notch (aN2) in quail neural tubes prevents the upregulation of RP markers. Left column represents electroporation of control GFP (a, g, m, s) ( $N=7$ for Bambi, and N=3 for Rspo1, Raldh2, and Draxin) or aN2-GFP (d, j, p, v) (N=7,15,6,6 for Rspol, Bambi, Raldh2, and Draxin, respectively). Middle column depicts ISH for the denoted genes in control GFP (b, h, $\mathbf{n}, \mathbf{t})$ or aN2 (e, $\mathbf{k}, \mathbf{q}, \mathbf{w})$, and right column is an overlay of the precedent, respectively. Note that control GFP-treated cells co-express both GFP and the RP genes (arrowheads), whereas aN2-treated cells are devoid of marker expression (arrows). Bar $=50 \mu \mathrm{m}$

\section{Loss of Notch results in absence of RP and dl1 interneurons}

It has been previously shown that in the absence of a $\mathrm{RP}$, development of dI1, the dorsal-most interneurons, is abnormal [41, 42]. We examined whether Notchdependent failure of RP formation is associated with a similar phenotype. Math1-positive dI1 interneuron progenitors were located lateral to the RP in E10.5 and E11.5 embryos. In contrast, in Wnt1-Cre; Mibl ${ }^{f l / f l} \mathrm{em}$ bryos, very few Math1+ progenitors remained at E10.5 and none were detected at later ages (Fig. 8a-d). Hence, in the absence of a RP, the formation of dI1 interneurons is compromised.
Next, we monitored expression of Ngn1+ and Ngn2+ dI2 interneuron progenitors and observed that their localization in the mutants progressively shifted towards the dorsal midline at the expense of the missing dI1 population. Although ISH of these markers prevented us from accurate quantification, a clear increase in the dI2 interneuron subset was apparent at both stages (Fig. 8e-l), suggesting aberrant specification of dorsal progenitors into dI2 neurons. Notably, Isl1+ dI3 interneurons were also shifted dorsal-ward in Wnt1-Cre; $M i b 1^{f l f l}$ embryos, but their total amount was unaffected (Fig. $8 \mathrm{~m}-\mathrm{p}, \mathrm{y}$ ). Further visualization of AP2 $\alpha$-positive

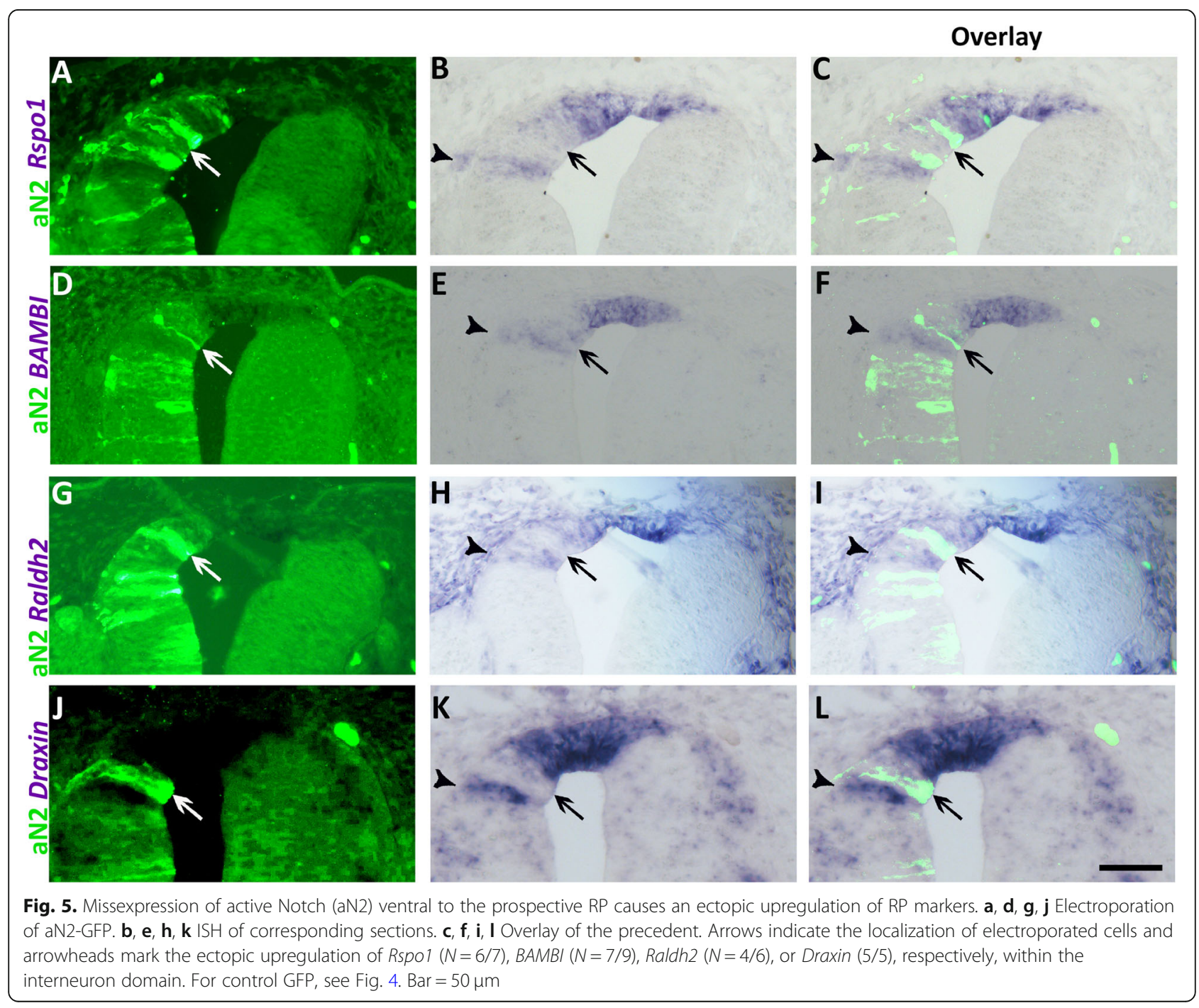



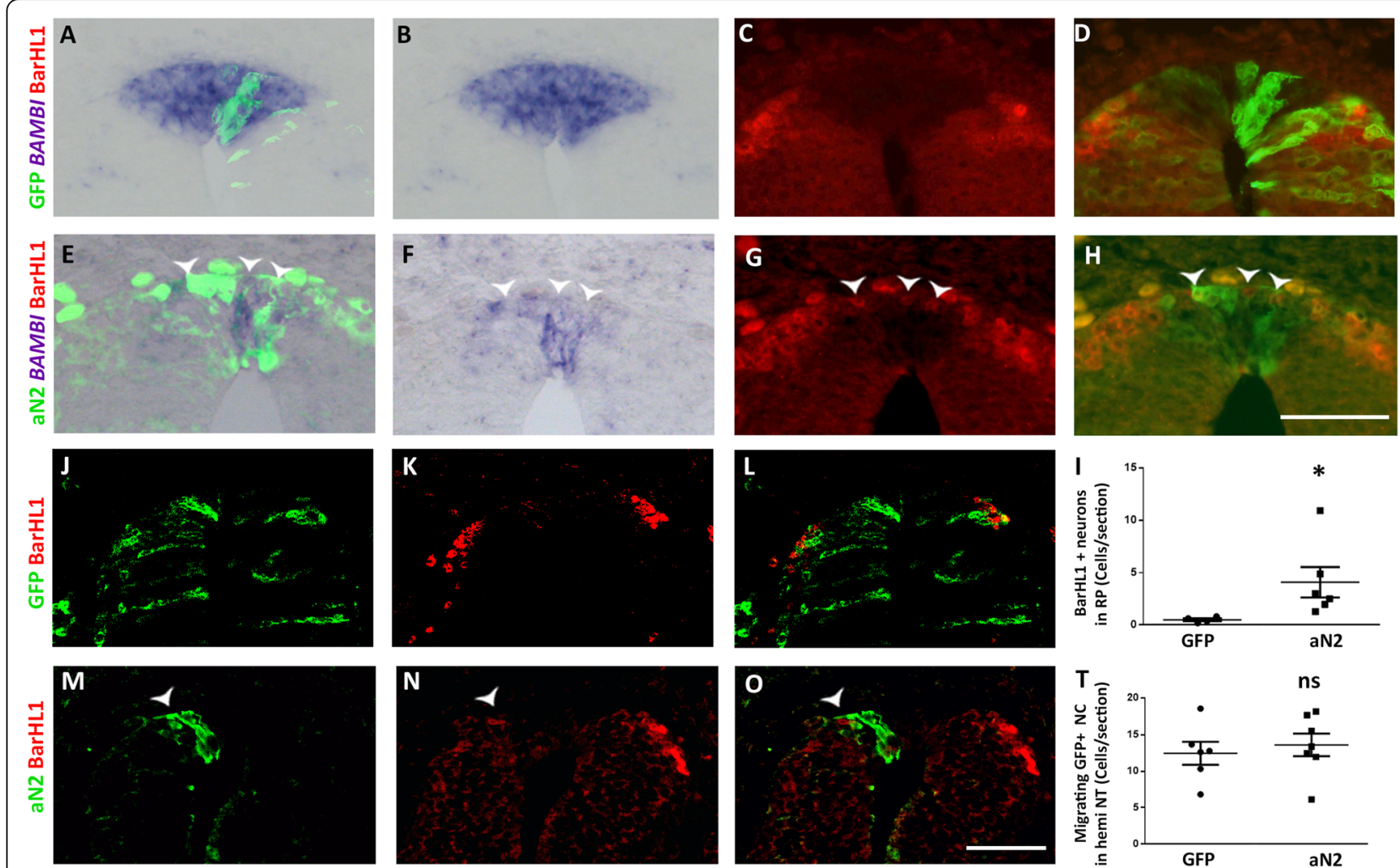

GFP HNK1
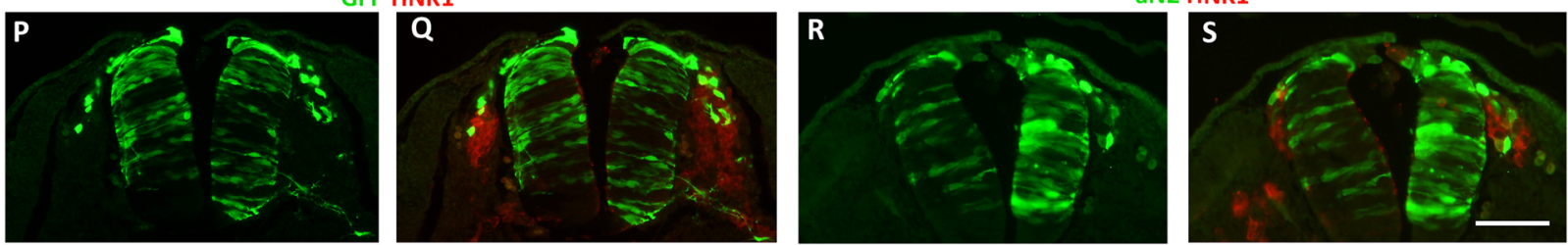

Fig. 6. Missexpression of active Notch induces differentiation of dl1 interneurons at the expense of RP traits, yet has no effect on early NC development. a-d Electroporation of control GFP. $\mathbf{e}-\mathbf{h}$ Electroporation of aN2-GFP. Note that control GFP has no effect on BAMBI mRNA expression $(\mathbf{a}, \mathbf{b})$ whereas its upregulation is compromised upon aN2 transfection $(\mathbf{e}, \mathbf{f})$. No BarHL1-positive dl1 interneurons are apparent in the RP of control embryos $(\mathbf{c}, \mathbf{d})(N=4)$. In contrast, numerous BarHIL-positive interneurons are present in the RP of aN2-treated cases $(\mathbf{g}$, $\mathbf{h}$, arrowheads). ( $N=6)$. i Quantification of the number of BarHL1+ interneurons/section present in the RP $\left(N=6\right.$ for both control and aN2, $\left.{ }^{*} p<0.02\right)$. j-o Electroporation of control GFP (j-I) or aN2 ( $\mathbf{m}$ - $\mathbf{o})$ followed by immunolabeling with BarHL1 antibody. Note that control GFP had no effect on interneuron development $(N=4)$ whereas no BarHL1+ interneurons were apparent in aN2-treated tubes $(N=6$, arrowhead). p-s Gain of Notch activity has no effect on NC migration. Electroporation of control GFP or aN2 at 22ss followed 1 day later by immunostaining for GFP (green) and the NC marker HNK-1 (red). Note in both cases the migration of GFP+/HNK1+ NC cells. $\mathbf{t}$ Quantification of the number of GFP-labeled migrating NC cells per section from hemi-neural tubes of control $(N=6)$ and aN2-treated $(N=7)$ embryos $(p=0.6, n s)$. Bar $=50 \mu m$

dI2-5 interneurons [40], revealed a similar dorsal shift at both stages, with their total amount being significantly enhanced in Wnt1-Cre; Mibt fl/fl embryos at E10.5 and E11.5, respectively (Fig. 8q-t, z). Together, this increase is likely to be accounted for by expansion of the dI2 population at the expense of dI 1 interneurons, since the Islet1+ dI3 interneurons were not quantitatively affected. Consistent with the replacement of RP by interneurons, we further documented a remarkable dorsalization of the neuronal marker Tuj1 in Wnt1-Cre; Mib1 ${ }^{f l / f l}$ embryos that was already apparent at E10.5 and progressively reached the dorsal midline by E11.5 (Fig. 8u-x).
As also previously reported, we observed no effect on specification and emigration of AP2 $\alpha+\mathrm{NC}$ cells at E9.5 in Wnt1-Cre; Mib1 fl/fl mutants when compared to wildtype ((Additional file 12: Fig. S10 A,B, and see [37] for quantification), suggesting that Notch signaling is involved in RP/interneuron development but not in early stages of $\mathrm{NC}$ ontogeny, as also seen in our quail experiments.

In addition, we asked whether the loss of RP and dI1 interneurons was due to enhanced cell death. No evidence for cleaved caspase3-positive cells was found in the dorsal NT of Wnt1-Cre; Mib1 $1^{f l f l}$ embryos at E9.5, 


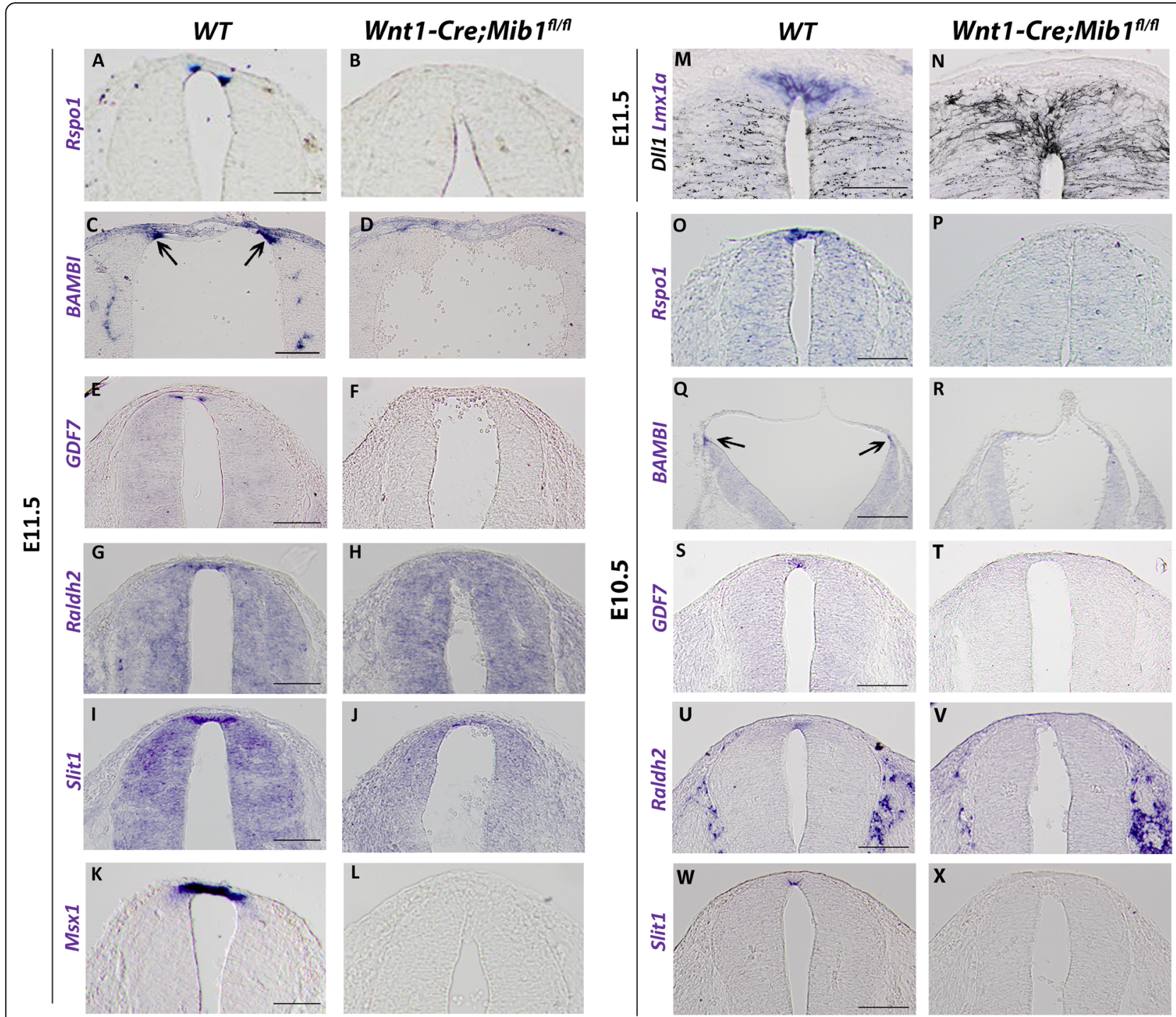

Fig. 7. Lack of roof plate formation in the absence of Notch signaling. In situ hybridization of definitive RP markers on transverse sections

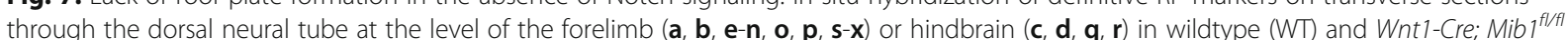
mouse embryos at E11.5 and E10.5. Expression of all RP in situ hybridization markers are missing from mutants at E11.5 and E10.5: Rspol (a, b and

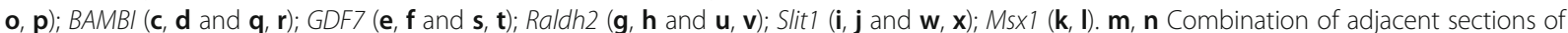
Lmx1a in situ hybridization (blue) and immunostaining for DII1 (black) showing complementary expression of the two markers at E11.5 in WT embryos but loss of Lmx1a in mutants with a corresponding dorsal expansion of Dll1. Bar $=100 \mu \mathrm{m}$

E10.5, or E11.5 when compared to control embryos. In contrast, NC-derived dorsal root ganglia revealed significant cell death at E11.5, as previously documented (Additional file 12: Fig. S10, A-F) [37]. Likewise, we tested for cell proliferation of the Dll1-negative dorsal cell population by pulse labeling E10.5 wildtype and Wnt1-Cre; $M i b f^{f l / f l}$ embryos with EdU. About $40 \%$ of prospective RP progenitors still proliferated at this stage in wildtype embryos. A 30\% reduction in the proportion of EdU+ nuclei was measured in the mutants compared to controls (Additional file 10: Fig. S10 G-I). Reduced proliferation in the dorsal NT of Wnt1-Cre; Mibr $1^{f l / f l}$ embryos at E10.5 coincides with the replacement of RP progenitors with post-mitotic interneurons (Figs. 7 and 8).

Altogether, in the absence of Notch signaling, initial NC development was normal, yet no RP or dI1 interneurons developed. This loss could not be explained by changes in cell death or proliferation; instead, it is likely to be accounted for by aberrant specification of the dorsal NT with corresponding expansion of the dI2 interneuron population.

\section{Discussion}

Understanding the role/s of gene activity in the transition between cellular states and fate decisions is essential 


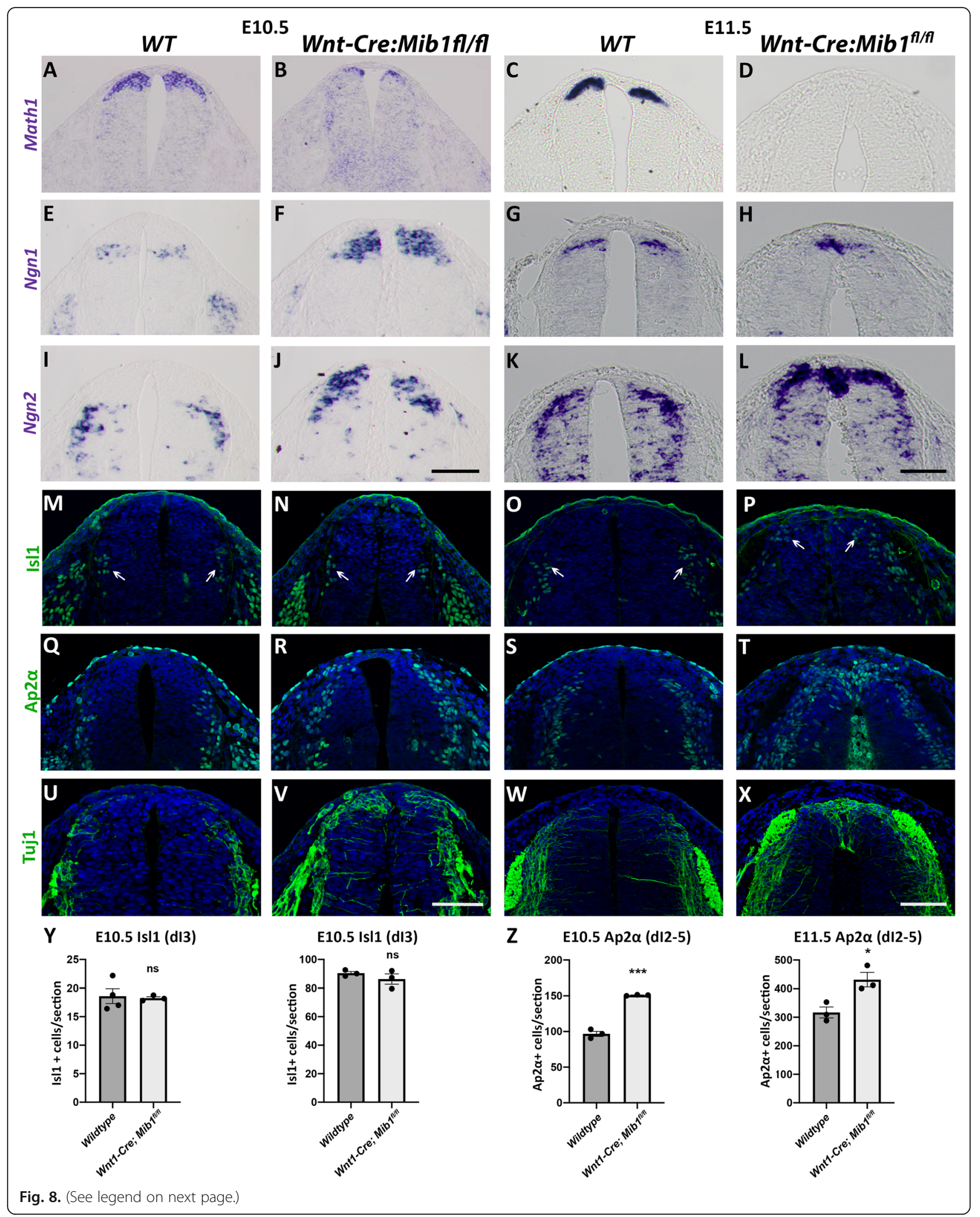


(See figure on previous page.)

Fig. 8. Re-specification of the dorsal neural tube domain in the absence of Notch signaling. a-I In situ hybridization of transverse sections through the neural tube at the level of the forelimb in wildtype (WT) and Wnt1-Cre; Mib $1^{f / f l}$ mouse embryos. Math1 expression at E10.5 (a, b) and E11.5 (c, d) showing loss of dl1 population in mutants; Ngn1 expression at E10.5 (e, $\mathbf{f})$ and E11.5 $(\mathbf{g}, \mathbf{h})$ and Ngn2 expression at E10.5 (i, j) and E11.5 (k, I) showing dorsal-ward expansion of the dI2 population. $\mathbf{m}-\mathbf{x}$ Immunostaining of transverse sections through the dorsal neural tube at the level of the forelimb in wildtype (WT) and Wht1-Cre; Mib ${ }^{f / f l}$ mouse embryos. Isl1 expression at E10.5 (m, $\left.\mathbf{n}\right)$ and E11.5 (o, p) showing a dorsalward expansion of dl3 interneurons at E11.5 (arrows); AP2a expression at E10.5 (q, r) and E11.5 (s, t) showing a dorsal-ward expansion in mutants at both stages (positive staining within the neural tube lumen in T is due to erythrocyte autofluorescence); Tuj1 expression at E10.5 (u, v) and E11.5 (w, x) showing ectopic expression of the axonal marker Tuj1 in the dorsal neural tube of mutants. $\mathbf{y}, \mathbf{z}$ Quantification of dl interneuron subtypes in the dorsal neural tube at E10.5 and E11.5. Isl1-positive dl3 interneuron numbers are unchanged in Wnt1-Cre; Mib 1/flfl embryos (y), whereas AP2a-positive dl2-5 interneuron numbers are increased in Wnt1-Cre; Mib $1^{f / f f l}$ embryos at both E10.5 and E11.5 (z). N=3-4 embryos; ns, not significant; ${ }^{*} p=0.022 ;{ }^{* * *} p=0.0001$. Bar $=50 \mu \mathrm{m}$

for elucidating mechanisms of normal development as well as the origin of embryonic malformations. The dorsal NT provides an excellent platform for investigating the sequential formation and delamination of a variety of NC cell types followed by the end of NC production/ emigration and the concomitant establishment of the definitive RP [1]. Although the formation and emigration of the NC has been extensively studied [2, 3, 43], how the dorsal NT moves from the early NC all the way into the definitive RP stage remains largely unknown. This is mainly due to the scarcity of RP-specific markers that enable tracking of the shift in cell identity and understanding the molecular pathways involved in the process. Using RNA-seq and ISH, we identify genes differentially expressed at either stage in the trunk level of the axis, their detailed expression patterns, and infer below on their possible functions in the above transition.

The observed coverage patterns correlate well with the known functions of BMP signaling in the proliferation, delamination, and emigration of NC cells [39, 44] and, in particular, with the reported end of BMP activity that leads to the arrest of $\mathrm{NC}$ emigration and $\mathrm{RP}$ formation [5]. The latter process is illustrated by the upregulation of the BMP inhibitors BAMBI, Grem1, Norrin, Smad3, and PRDM12 and also that of HES4, the quail ortholog of Hairy1/HES1, shown to inhibit BMP signaling in RP [5]. Furthermore, synthesis of factors of the BMP family shown to be necessary for adjacent interneuron development, such as Gdf7 [10], or BMP4 and BMP7 [9, 13], is either upregulated or maintained in RP, respectively. Hence, we further extend the molecular repertoire in support of the notion that the RP becomes refractory to BMP signaling whereas interneurons continue responding to this family of RP-derived factors.

Interestingly, the observed upregulation of the BMP inhibitor $B A M B I$ raises the possibility that the retinoic acid (RA) pathway is also involved in the end of NC production and/or in RP formation. First, BAMBI is a direct target of RA [45]; second, the RA biosynthetic enzyme Raldh 2 begins to be transcribed in RP in addition to its earlier synthesis in paraxial mesoderm (Fig. 2) and so are $C R A B P 1$ that binds and mediates RA activities (Fig. 2)
[46] and the RA modulator Znf536 [47]. Together, we posit that RA emanating from the RP may contribute to the end of NC production by inhibiting BMP signaling, at least partially through BAMBI, a hypothesis that will be put to the test.

One of the major genes produced selectively in RP but not premigratory $\mathrm{NC}$ is $R$-spo1. All four secreted Rspondins are unique agonists of Wnt-mediated signaling [48] which plays a fundamental role in G1/S transition of NC cells and their delamination [44], yet whose possible function in RP formation was so far not explored. R-spondins bind to Lgr 4/5/6 receptors and bring these into position to interact with the ubiquitin ligases Rnf43/Znrf3. This interaction results in membrane clearance of the latter and consequent persistence of the Wnt/Frizzled/Lrp complexes in the plasma membrane, enhancing Wnt signal strength and duration [49, 50]. Rspo1 was reported to be involved in male sex reversal and adult intestinal stem cell homeostasis [48], yet its role in RP development remains unknown. Since its expression in RP was compromised in $W n t 1 / 3 a$ double knockout mice, it was suggested that it may contribute to the development of dorsal NT under the regulation of Wnts [27]. Consistent with such a possibility, our RNAseq unravels the upregulation of the R-spo1 receptor Lgr5, induction of Norrin, a R-spo/Wnt agonist that additionally inhibits BMP activity [51], and a decrease of Rnf43. Moreover, several Wnt inhibitors are downregulated in RP, such as Dkk1, Frzb,DACT2, and Sfrp2. In contrast, the Wnt receptors $F z 3$ and $F z 10$, the downstream target Axin2, and the co-receptors $L R P$ 4,8, and 11 are enhanced.

Our data show that $R$-spo1, Gdf7, and CRBP1 are predominantly expressed in the lateral RP along the trunk (our data and see [18, 27]) abutting the boundary between the RP and adjacent neuroepithelium. In contrast, Axin2, Lgr4, and Rnf43, all belonging to the R-spo/Wnt module (see above), are expressed in the medial RP, suggesting that the trunk RP is molecularly heterogeneous. Indeed, this molecular heterogeneity also seems to be conserved in mice where $R$-spol also marks the lateral RP domains. Apart from uncovering molecular 
components of this heterogeneity in the trunk, we confirm the notion suggested for the hindbrain RP that the latter is formed by molecularly and temporally separable lineages $[[17,18]$, and refs. therein]. Importantly, at cranial levels, only the lateral RP seems to contribute to the formation of the choroid plexus [17]. Whether the observed heterogeneity in the trunk RP predicts a differential fate and/or function of medial vs. lateral domains remains to be elucidated.

Taken together, results of our transcriptome data enabled us first to postulate new roles and interactions between candidate signaling pathways and specific markers of RP domains in the dynamics of dorsal NT development, and second, to plan concrete experiments to put these to the test.

Along this line, we presently addressed the role of the highly conserved Notch signaling pathway in RP formation. In both avians and mice, the dorsal limit of Notch ligand expression corresponds to the ventral boundary of the RP. Although Notch mRNAs are widely transcribed, analysis of N1ICD reveals a similar dorsal limit of activity as that of its ligands and of its modulator LNFG. This suggests that the ventral border of the prospective RP, where all the components of the pathway meet, is a region of Notch/Dll signaling.

Mosaic electroporation of active Notch in prospective $\mathrm{RP}$ prevented the advent of RP-specific markers in a cell-autonomous manner, while inducing them in ectopic positions immediately ventral to the RP in a noncell-autonomous manner. Reciprocally, loss of endogenous Notch activity prevented de novo RP formation. Hence, both gain and loss of function approaches unravel a role for Notch signaling in development of the definitive RP.

This interpretation was possible by implementing RPspecific markers, not expressed in the earlier premigratory NC population. Previous studies used BMP4, Wnt1, and $L m x 1$ as RP markers [52], yet these are transcribed in both populations.

Of importance is the finding that gain of Notch function in the dorsal NT prevented the expression of endogenous RP traits, while upregulating ectopically specific markers of dI1 interneurons. Conversely, loss of RP development in the absence of Notch signaling compromised development of Math1+ dI1 interneurons, a phenotype that was coincident with expansion of the dI2 population (Fig. 9). Likely, this may be associated with our observation that Notch activity in normal mouse embryos corresponds to the dorsal limit of dI1 interneurons. These results show, first, that Notch plays a role in the binary choice between RP and interneuron fates, and second, confirm that the RP is necessary for at least dI1 interneuron development [41, 42]. Alternatively, changes in Notch activity in the boundary between RP and interneurons may affect both populations altogether (Fig. 9f), as previously suggested for the hindbrain where a well-defined boundary zone may act as a bidirectional signaling center [18].

In this regard, Notch may act to create an interface between RP and non-RP cell types. This is consistent with missexpression of active Notch in non-RP domains, where it downregulates interneuron identity cell autonomously and induces RP traits in adjacent cells. Similar effects were reported on the formation of interhombomeric boundaries in zebrafish [53], and in cultured mouse neural progenitors transfected with the Notch target Hes1 [54]. Thus, forced expression of Notch in non-RP cells prevents them from differentiating into neurons while inducing RP properties in neighboring progenitors. Reciprocally, loss of Notch function prevented RP formation and the dorsal NT became instead populated by dI2 interneurons (Fig. 9). Our results are in agreement with the phenotypes observed in triple Hes1,3,5 mutants, in which the RP at spinal cord levels was compromised and replaced by ectopic interneurons [54]. Hence, our loss of function data are consistent with our gain of function results. Both can be explained by the lack of formation of a proper border between RP and non-RP cells in combination with the antineurogenic role of Notch.

Notably, activities of Notch may be context-dependent as, in the dorsal-most NT, where no endogenous Notch activity is detected, transfection of active Notch prevented the transcription of endogenous RP markers (Fig. 9). This is consistent with missexpression of LNFG that repressed formation of the zona limitans intrathalamica, another boundary structure similar to the RP that is devoid of endogenous LFNG [55]. Together, our gain of function results suggest that cells overexpressing Notch activity cannot become RP, yet are able to induce RP characteristics non-cell autonomously.

The above results further exemplify the complexity of Notch signaling. Our finding showing the non-cellautonomous induction of RP next to cells transfected with active Notch could also be consistent with non$R P$ cells inducing a $R P$ fate. Although the $R P$ is known to function as a signaling center, reciprocal interactions could exist between RP and non-RP (Fig. 9). These might not be necessarily mediated by Notch itself, as Notch activity seems to be confined to the non-RP area. In this regard, Dll-Notch signaling was shown to indirectly induce cranial NC by the activation of BMP. Moreover, Notch simultaneously restricted the lateral margin of the cranial $\mathrm{NC}$ territory by cell-autonomously repressing the $\mathrm{NC}$ fate [56]. This provides an example of bimodal functions of Notch signaling in the regionalization of distinct domains. 

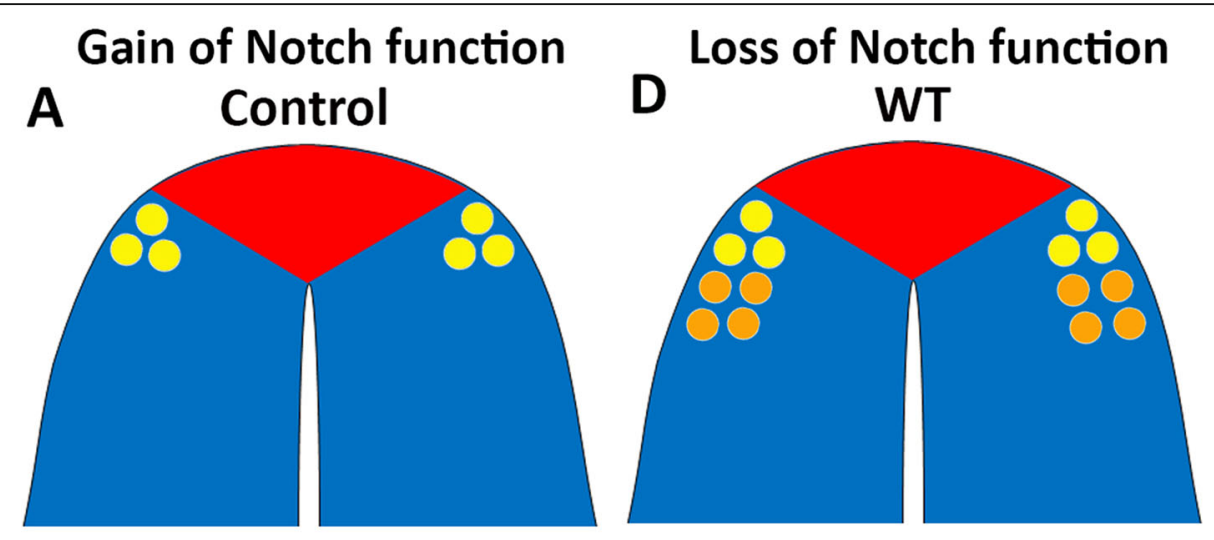

B aNotch in RP
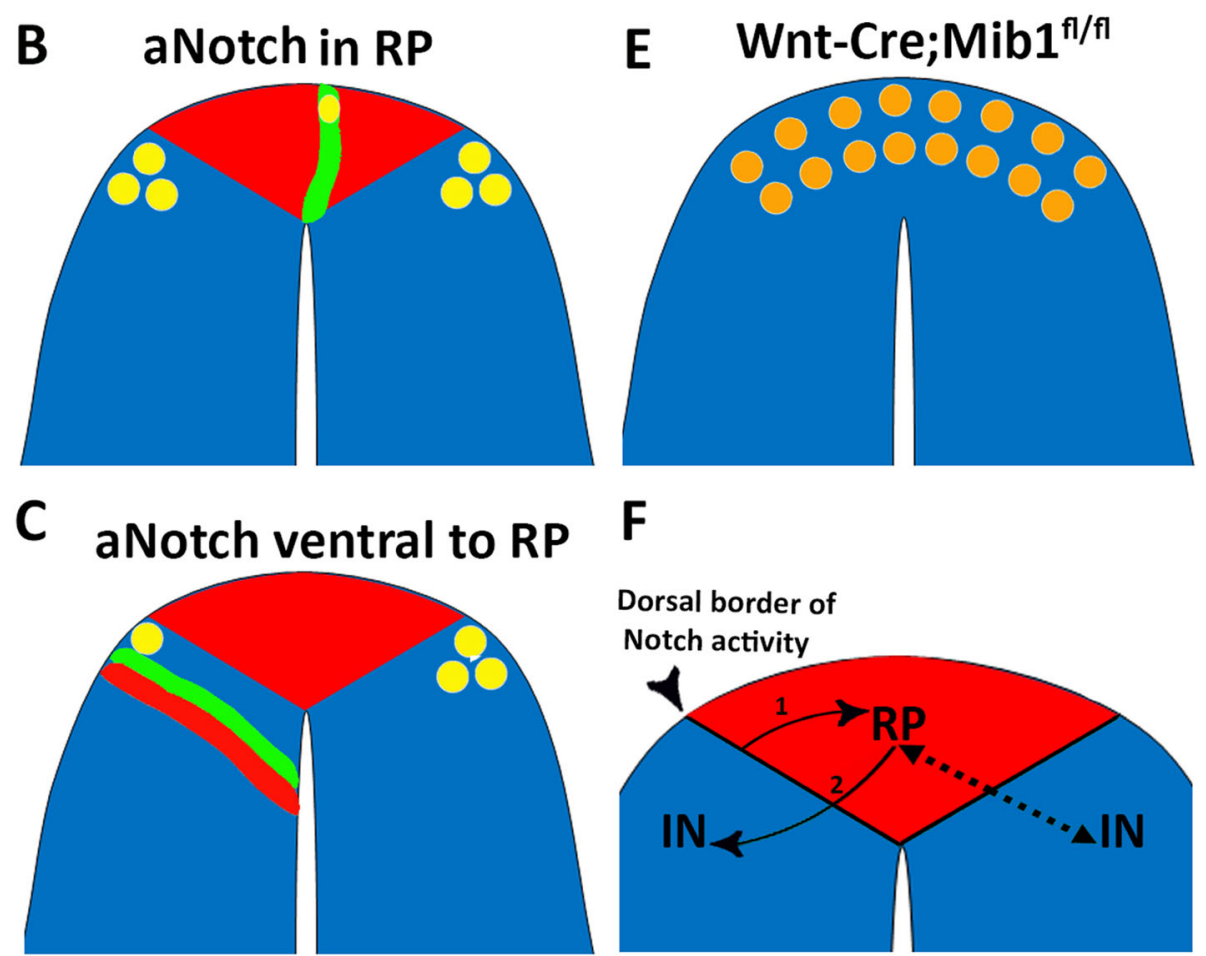

\section{Dorsal border of} Notch activity

\section{RP}

Fig. 9. Role/s of Notch signaling in RP/interneuron development. a-c Gain of Notch activity in quail embryos. In controls (a), the RP (red) is flanked by adjacent dorsal interneurons (dl1, yellow). b Missexpression of active Notch (green) in RP prevents expression of RP-specific traits in a cell-autonomous manner while upregulating BarHL1, a marker of dl1 interneurons. c Missexpression of active Notch ventral to the RP suppresses dl1 interneuron fate while upregulating RP markers in adjacent cells. $\mathbf{d}$, e Wild type (WT) mouse embryo showing the RP flanked bilaterally by dl1 and $\mathrm{dl} 2$ interneurons (d). Loss of Notch function in Wnt1-Cre; Mib1 mutants prevents the development of RP and of dl1 interneurons, with a corresponding dorsal expansion of the dl2 interneuron population ( $E$, orange). $\mathbf{f}$ At the trunk level of the axis, a sharp boundary between non-RP (blue) and RP (red) is demarcated by the dorsal extent of Notch activity (arrowhead). Notch signaling from this domain acts either directly or indirectly to induce RP formation (arrow 1 in left side of the image) and the RP in turn induces dorsal interneurons (arrow 2, IN). Alternatively, this boundary acts bidirectionally to induce both RP and INs (dotted arrows, right side). See text for detailed discussion

We cannot rule out the possibility of a timedependent activity of Notch in the present context. It is possible that initially, Notch signal coming from Dll1expressing cells lateral to the NC/RP domain instructs dorsal NT cells to adopt a RP fate. This would be consistent with our previous results showing that both NC and RP are generated from common Foxd3-positive precursors [26], with the RP cells stemming from the lateral-most domain $[25,26]$. Subsequently, Notch signal in non-RP cells may maintain their non-RP identity (e.g., dorsal interneuron fate) and set the interface between $\mathrm{RP}$ and non-RP. 
It is noteworthy that different experimental paradigms affecting the advent of a definitive RP have varied outcomes on dorsal interneurons. For instance, ablation of the RP by targeting diphtheria toxin under the regulation of Gdf7 caused the disappearance of both dI1 and dI2 interneurons and the dorsal midline was occupied instead by dI3 cells [42]. In $L m x 1 a$ mutants, however, Math1+ dI1 interneurons were generated albeit in reduced numbers and no effect on dI2 was monitored [41]. This difference in phenotype severity could be accounted for by the documented lack of Gdf7, BMP6, Msx1, and Wnt1 in the Gdf7 mutants compared with the Dreher mice in which residual expression of Wnt1 and Msx1/2, factors shown to operate on interneuron development persisted [52, 57].

In our loss of Notch function experiments, dI1 progenitors, as assessed by Math1 expression, were reduced albeit still apparent at E10.5, and absent by E11.5; dI2, but not dI3 interneurons, were expanded in their absence. It is possible that early specification of interneuron subsets occurs prior to the advent of the definitive RP by dorsal NT-derived BMPs [9, 12, 58]. Additionally, it is possible that BMPs derived later from the ectoderm dorsal to the spinal cord [59] still enable development of selected interneurons of the dI2 subclass, expanded in our mutants. In this context, it would be interesting to examine the relationship between Notch signaling and dorsal NT factors of the BMP and Wnt families.

In both avians and mice, the protocols implemented did not affect early development of NC cells. In avians, epidermal Dll1 signaling was shown to induce cranial NC cells indirectly via BMP activation [56]. In the present study, gene missexpressions were performed following initial NC induction, suggesting that Notch plays no role on specified premigratory $\mathrm{NC}$ or during migration. Nevertheless, at later stages, Notch was shown to play significant roles in neuron-glia fate decisions within NC-derived dorsal root ganglia $[37,60]$.

\section{Conclusions}

Taken together, the combination of RP-specific markers with targeted gene misexpression allows us to begin understanding the mechanisms underlying segregation of the three sequential lineages that inhabit the dorsal NT, e.g., $\mathrm{NC}, \mathrm{RP}$, and interneurons. Of the precedent lineages, we report that Notch signaling is required for RP formation and for the choice between RP and interneuron fates, yet is dispensable for early stages of $\mathrm{NC}$ ontogeny.

\section{Methods}

\section{Quail and mouse embryos}

Fertilized quail (Coturnix coturnix japonica) eggs were obtained from commercial sources (Moshav Mata) and incubated at $38{ }^{\circ} \mathrm{C}$ to the desired developmental stages.
All experiments on mice were approved by the SA Pathology and University of South Australia Animal Ethics Committee (project \# u26/20). To obtain embryos of defined gestational ages, mice were mated in the evening, and the morning of vaginal plug formation was counted as embryonic day (E) 0.5. Pregnant dams were humanely euthanized at relevant days post vaginal plug detection by $\mathrm{CO}_{2}$ inhalation and cervical dislocation. To delete Mib1 specifically in the dorsal neural tube, we mated $\mathrm{Mibl}^{f l /+}$ males carrying a heterozygous Wnt1-Cre transgene to $M i b 1^{f l f l}$ female mice [61]. At least $3 \mathrm{em}-$ bryos/age/genotype were analyzed for each experiment.

\section{Expression vectors and in ovo electroporation}

For transcriptome analysis, pCAGGS-EGFP $(2-5 \mu \mathrm{g} / \mu \mathrm{l})$ was microinjected into the lumen of the NT opposite the segmental plate region of embryos aged 18ss to label the early premigratory $\mathrm{NC}$ or at the flank region of embryos aged 43ss to label the RP. For functional studies, electroporations were directed to the flank level (somites 20-25) of embryos aged 22-25 somites, as detailed in the "Results" section.

An inducible version of the intracellular part of Notch2 (aN2) [62] was sub-cloned into a tetracyclinesensitive plasmid (pBI-TRE-GFP) [63] as previously described [64]. Active cNotch-1 (pMIW-CNIC $\Delta$ c89) [65] was used as described [66]. pBI-TRE-GFP or pCAGGs were used as controls.

To perform focal electroporations directed to the dorsal NT at each stage, a 5-mm tungsten electrode was inserted under the blastoderm (negative electrode) and a fine 2-mm-long electrode was placed dorsal to the neuroepithelium. For hemi-NT transfections, electrodes were placed at both sides of the NT. A square wave electroporator (BTX, San Diego, CA, USA) was used to deliver one to three pulses of current at $10-20 \mathrm{~V}$ for $8 \mathrm{~ms}$.

\section{Cell dissociation}

Dorsally electroporated NT's were dissected manually 6-8 h after electroporation before significant emigration of NC cells begun in the early-stage embryos. Prior to NT isolation, embryos were inspected under a fluorescent binocular microscope. Embryos with emigrating cells, or with large electroporated domains were excluded. Single-cell suspensions were obtained by enzymatic digestion with $0.01 \%$ Trypsin $/ 0.02 \%$ EDTA and $7 \mu \mathrm{g} / \mathrm{ml}$ DNAse1 in $\mathrm{Ca} / \mathrm{Mg}$-free PBS for 3.5 and $4.5 \mathrm{~min}$ for $\mathrm{NC}$ and RP stages, respectively. Dissociation was completed by manual pipetting. The resulting cell suspensions were centrifuged at 1000 RPM in an Eppendorf centrifuge, washed once with washing solution $(\mathrm{Ca} / \mathrm{Mg}$ free PBS/10\%FCS/0.1 units of RNAse inhibitor), and then with fluorescent activated cell sorting (FACS) 
buffer (PBS CaMg-free /2\% FCS). The final suspension was filtered through a $40-\mu \mathrm{m}$ pore size Cell strainer.

\section{FACS sorting}

ARIA III (BD Biosciences) was used for sorting. The cut-off for sorting the electroporated GFP+ cells and for exclusion of dead/damaged PI+ cells was chosen manually. Three samples for each stage containing a total of 1000-6000 GFP+/ propidium iodide (PI)-negative cells were collected in Trizol. As expected from very focal transfections, the percentage of GFP+/PI- cells of the total population was about $0.3 \%$ at both stages. Total viability of the cells was $99.9 \%$ and at least $95 \%$ or $87 \%$ for the electroporated GFP+ cells at NC and RP stages, respectively.

\section{Library preparation}

RNA was extracted according to standard Trizol protocol (Invitrogen), using LPA as carrier (Sigma). Amount and quality were checked on Agilent Bioanalyzer using an RNA-pico chip. In total, 120 pg RNA of each sample was taken for library preparation using the CEL-Seq2 protocol [67], with the following modifications: RNA was added to dNTP/ERCC spike-in mix, and 3 different CEL-Seq primers added to each sample as technical replicates. Library was sequenced on HiSeq2500 in rapid mode, 15 bases for read 1, and 50 bases for read 2 .

\section{Bioinformatic analysis}

Demultiplexing was performed according to the pipeline from CEL-Seq2 [67], R2 reads were split into their original samples using the CEL-Seq barcode from R1. The reads were cleaned using trim_galore (uses cutadapt version 1.10) for removal of adaptors, polyA, low-quality sequences (Phred $<20)$, and short reads $(<25 \mathrm{bp}$, after trimming). Mapping to the Coturnix coturnix japonica genome was conducted using Tophat 2 version 2.1.0. The reference and annotation files were downloaded from https://www.ncbi.nlm.nih.gov/genome/term=Coturnix + japonica, ftp://ftp.ncbi.nlm.nih.gov/genomes/refseq/ vertebrate_other/Coturnix_japonica/latest_assembly versions/GCF_001577835.1_Coturnix_japonica_2.0, respectively. HTseq-count version 0.6 .1 was used for counting. Normalization and differential expression analysis using DESeq2 $\mathrm{R}$ package version 1.14.1 was performed after combining reads of the technical replicates.

\section{Proliferation assays and immunohistochemistry Quail embryos}

Incorporation of $\mathrm{BrdU}$ was performed $1 \mathrm{~h}$ before fixation and immunolabeling was done as described [5]. For immunostaining, embryos were fixed in $4 \%$ formaldehyde overnight at $4{ }^{\circ} \mathrm{C}$, processed for paraffin wax embedding, and sectioned at $8 \mu \mathrm{M}$. Antibodies used were rabbit anti
BarHL1 (1:100, Sigma, HPA004809), rabbit anti GFP (1: 1000, Invitrogen, Thermo-Fisher Scientific, A6455), and rabbit anti RFP (1:000, OriGene, AP09229PU-N). Nuclei were visualized with Hoechst.

\section{Mouse embryos}

To examine cell proliferation, EdU (5-ethynyl-2'-deoxyuridine) (Life technologies) was prepared in $0.9 \% \mathrm{NaCl}$ solution at $10 \mathrm{mg} / \mathrm{ml}$. EdU was delivered by intraperitoneal injections to pregnant dams at E10.5 at $100 \mathrm{mg} / \mathrm{kg}$ of body weight and embryos were collected $1 \mathrm{~h}$ after injection.

Embryos were fixed in $4 \%$ formaldehyde overnight at $4{ }^{\circ} \mathrm{C}$, cryopreserved in $20 \%$ sucrose, and embedded in OCT compound for cryosectioning. Sections were blocked in 10\% Dako serum-free blocking reagent in PBS $/ 0.1 \%$ TritonX-100, followed by incubation in primary antibody for $2 \mathrm{~h}$ at room temperature or overnight at $4{ }^{\circ} \mathrm{C}$. Fluorescent Alexafluor-conjugated secondary antibodies were incubated for $1 \mathrm{~h}$ at room temperature. Sections were mounted in Prolong Diamond antifade with DAPI. The following primary antibodies were used: mouse anti-Tuj1, 1:750 (Sigma-Aldrich T5076); mouse anti-Isl1, 1:50 (DSHB 40.3A4); rabbit anti-cleaved Caspase-3, 1:500 (Cell Signaling Technology 9661); mouse anti-AP2 $\alpha, 1: 20$ (DSHB 3B5); sheep anti-Dll1, 1: 200 (R\&D Systems AF5026); rabbit anti-N1ICD, 1:100 (Cell Signaling Technology 4147). Sections of EdUlabeled embryos were incubated with Click-iT EdU Kit Alexa-555 conjugated (Life Technologies) following staining with primary antibodies.

\section{Antigen retrieval}

Immunostaining for interneuron markers and N1ICD required antigen retrieval. Paraffin-sectioned embryos were immersed in pre-boiled sodium citrate $\mathrm{pH}$ 6, 0.05\% Tween20, and microwaved for $10 \mathrm{~min}$ (20\% strength). Blocking was performed with $5 \%$ newborn calf serum (Biological Industries 04-102-1A, Israel) in PBS for $1 \mathrm{~h}$ at room temperature. Cryosections were incubated in $10 \mathrm{mM}$ sodium citrate $\mathrm{pH} 6.0$ at $90{ }^{\circ} \mathrm{C}$ for $20 \mathrm{~min}$, and then cooled to room temperature before proceeding with immunohistochemistry procedure as above.

\section{Tyramide amplification}

Immunostaining for N1ICD required tyramide signal amplification, performed using Invitrogen Alexa Fluor 555 Tyramide SuperBoost Kit (B40923). Following primary antibody incubation, sections were incubated with goat anti-rabbit horseradish peroxidase, then tyramide signal amplification was performed for $30 \mathrm{~min}$, following the manufacturer's instructions. 
In situ hybridization

\section{Quail embryos}

ISH was performed as described previously [36]. Embryos were fixed in Fornoy (60\% ethanol, 30\% formaldehyde, $10 \%$ acetic acid), then dehydrated in ethanol/ toluene, processed for paraffin wax embedding, and sectioned at $10 \mu \mathrm{m}$. Slides were sequentially rehydrated in toluene/ethanol/PBS, treated with proteinase $\mathrm{K}(1 \mu \mathrm{g} / \mathrm{ml}$, Sigma-Aldrich P2308) at $37^{\circ} \mathrm{C}$ for $7 \mathrm{~min}$, and then fixed in $4 \%$ formaldehyde at room temperature for $20 \mathrm{~min}$. Next, slides were washed in PBS followed by $2 \times$ SSC and hybridized in hybridization buffer $(1 \times$ salt solution composed of $2 \mathrm{M} \mathrm{NaCl}, 0.12 \mathrm{M}$ Tris, $0.04 \mathrm{M} \mathrm{NaH}_{2} \mathrm{PO}_{4} 2 \mathrm{H} 2 \mathrm{O}$, $0.05 \mathrm{M} \mathrm{Na}_{2} \mathrm{HPO}_{4}, 0.05 \mathrm{M}$ EDTA, pH 7.5), 50\% formamide, $10 \%$ dextran sulfate, $1 \mathrm{mg} / \mathrm{ml}$ Yeast RNA, and $1 \times$ Denhardt solution containing $1 \mu \mathrm{g} / \mathrm{ml}$ DIG labeled RNA probes (prepared with a DIG RNA labeling mix, Roche, 11277073910 ) for overnight at $65^{\circ} \mathrm{C}$ in a humid chamber. Post-hybridization, slides were rinsed in a rotating incubator with $50 \%$ formamide, $1 \times \mathrm{SSC}$, and $0.1 \%$ Tween 20, until coverslips dropped, and then an additional wash for $1 \mathrm{~h}$ followed by 2 washes in MABT $(10 \%$ Maleic acid $1 \mathrm{M} \mathrm{pH} 7.5,3 \% \mathrm{NaCl} 5 \mathrm{M}, 0.1 \%$ Tween 20) and preincubation in MABT/ $2.5 \%$ FCS. Anti-DIG-AP antibody (1/1000, Roche 11093274910) diluted in $\mathrm{MABT}+2 \% \mathrm{BBR}+20 \% \mathrm{FCS}$ was then added for overnight at room temperature. This was followed by rinsing in MABT and then in NTMT $(2 \% \mathrm{NaCl} 5 \mathrm{M}, 10 \%$ Tris $\mathrm{HCl}$ $1 \mathrm{M} \mathrm{pH} 9.5,5 \% \mathrm{MgCl} 21 \mathrm{M}, 0.1 \%$ Tween 20), and then incubation in NTMT + 1:200 NBT/BCIP Stock Solution (Sigma-Aldrich, 11681451001 ) at $37^{\circ} \mathrm{C}$ until the AP reaction was completed.

The following probes were kindly provided: Foxd3 [68, 69], Snail2 [70], Dact2 [71], Sfrp2, Dkk1, LFNG, and Wnt4 [72]. Additional probes were produced by PCR amplification (using Q5 high fidelity DNA polymerase) with specific primers (see Additional file 13: Table S3 for list of primers). Template for PCR was cDNA, which was synthesized by RNA precipitation followed by reverse transcription PCR. RNAs were produced from 20ss-E4 quail embryos. Tissue samples were homogenized with TriFast reagent, and RNA was separated with chloroform and isopropanol.

Importantly, all hybridizations, whether done in intact embryos at NC vs. RP stages, or in control vs. experimental embryos, were always performed for similar signal developing times per given probe and in the same experiment.

\section{Mouse embryos}

Section ISH was performed as described [73]. Riboprobes were transcribed from plasmids containing PCR amplified cDNA sequences (Additional file 13: Table S3). $\mathrm{mNgn1}, \mathrm{mNgn2}$, and $M s x 1$ riboprobes were kindly provided by F. Guillemot. These and Math1 were prepared as previously described $[37,74]$.

\section{Data analysis and statistics}

The number of Isl1+ or AP2 $\alpha$ + nuclei in the dorsal NT was monitored in at least 5 sections per wildtype or Wnt1-Cre; Mib1 ${ }^{f l f l}$ embryos. The number of BarHl1positive interneurons or of migrating GFP-positive NC cells was measured in at least 10 sections per embryo from control GFP or aN2-electroporated embryos. Graphs represent mean number of positive cells/section \pm SEM.

For measurement of BrdU incorporation, cells in 15 sections per embryo were counted and expressed as percentage of BrdU+/total GFP+ cells in the dorsal NT.

For monitoring cell proliferation in mice, the number of Edu-positive cells was counted in nuclei located dorsal to the boundary of Dll1 expression, i.e., Dll1-negative cells, and expressed as a percentage of total Dll1negative cells. At least 5 sections per embryo were quantified. Graphs represent mean number of positive nuclei \pm SEM.

Images were photographed using a DP70 (Olympus) cooled CCD digital camera mounted on a BX51 microscope (Olympus). For figure preparation, images were exported into Photoshop CS6 (Adobe). If necessary, the levels of brightness and contrast were adjusted to the entire image and images were cropped without color correction adjustments or $\gamma$ adjustments. Data were analyzed using unpaired Student's $t$ test. A $p$ value $\leq$ 0.05 was considered significant.

\section{Supplementary Information}

Supplementary information accompanies this paper at https://doi.org/10. 1186/s12915-021-01014-3.

Additional file 1: Fig. S1. Labeling of the dorsal NT at NC and RP stages followed by FACS analysis. Focal electroporations of GFP-DNA were directed to the dorsal NT at NC and RP stages. Embryos were sacrificed 6-8hr later to isolate premigratory NC progenitors prior to the onset of EMT. $(A, E)$ dorsal views of live embryos showing restricted labeling of the dorsal midline domain at both stages. (B,F) Focal dorsal labelings confirmed in transverse sections. Green cells outside the NT in F represent autofluorescence of blood cells. $(C, D, G, H)$ FACS purification of GFPlabeled cells. Note absence of GFP+ cells in control non-electroporated samples ( $C$ and $\mathrm{G}$ ) and very low proportions of propidium iodide $(\mathrm{PI})+$ dead cells in both control and electroporated cases. PI+ cells were excluded by gating and GFP+/PI- cells were collected $(D, H)$. As expected from focal transfections, the percentage of live, labeled cells (GFP+/PI-) was $0.32 \%$ and $0.28 \%$ of the total input for NC and RP, respectively. Abbreviations, EP, electroporation, FITC, fluorescein isothiocyanide. Bar= $50 \mu \mathrm{m}$.

Additional file 2: Table S1. A list of transcripts downregulated in RP when compared to NC. Excel file containing the list of transcripts downregulated in RP when compared to the premigratory NC stage. Based on triplicate samples for each stage. See Methods for technical details.

Additional file 3: Table S2. A list of transcripts upregulated in RP when compared to NC. Excel file containing the list of transcripts enriched in 
the RP when compared to the premigratory NC stage. Based on triplicate samples for each stage. See Methods for technical details.

Additional file 4: Fig. S2. In situ hybridization (ISH) for selected genes downregulated in RP compared to NC. (A, D, G, J, M) Transcripts expressed in the dorsal tube at the NC but not RP stage $(B, E, H, K, N)$. (C, $F, I, L, O)$ Quantification of gene expression levels stemming from the transcriptome analysis. NC1-3 and RP1-3 represent experimental triplicates. Note positive correlation between ISH and transcriptome results. In each chart, the $\mathrm{B}-\mathrm{H}$ adjusted $p$ value is indicated. Abbreviations, DRG, dorsal root ganglion, Es, epithelial somite, NT, neural tube, No, notochord. Bar $=50 \mu \mathrm{m}$

Additional file 5: Fig. S3. ISH for selected genes expressed in the RP as well as in more ventral domains of the NT. (A, D, G, J) ISH at NC and RP $(B, E, H, K)$ stages, respectively. (C,F,IL) Quantification of gene expression levels stemming from the transcriptome analysis. In each chart, the B-H adjusted $p$ value is indicated. Note at RP stage the extended expression of transcripts beyond the RP domain. Abbreviations, NT, neural tube, No, notochord. Bar $=50 \mu \mathrm{m}$

Additional file 6: Fig. S4. ISH for selected "non-RP" genes upregulated at the RP stage but not transcribed in RP. $(A, D, G, J, M, P, S) I S H$ at the NC stage. $(B, E, H, K, N, Q, T) I S H$ at the RP stage. (C,F,I,L,O,R,U) Quantification of gene expression levels. In each chart, the $\mathrm{B}-\mathrm{H}$ adjusted $\mathrm{p}$ value is indicated. Note in the middle column, the expression of transcripts throughout the NT except for the RP in spite of transcript levels being upregulated in RP compared to NC (right column). Bar $=50 \mu \mathrm{m}$

Additional file 7: Fig. S5. ISH of adjacent sections with selected RP markers in combination with the "non-RP" marker lunatic fringe (LFNG). $(A, D, G, J, M, P, S) I S H$ for RP markers. (B,E,H,K,N,Q,T) ISH for LNFG. (C,F,I,L,O,R,U) Overlay of the precedent showing complementary marker expression. $\mathrm{Bar}=50 \mu \mathrm{m}$.

Additional file 8: Fig. S6. ISH of adjacent sections with selected "non$\mathrm{RP}^{\prime \prime}$ genes in combination with Rspo-1. (A,D,G,J,M) ISH for non-RP markers. $(\mathrm{B}, \mathrm{E}, \mathrm{H}, \mathrm{K}, \mathrm{N}) \mathrm{ISH}$ for Rspo 1. (C,F,I,L,O) Overlay of the precedent showing complementary marker expression. $(P, Q, R)$ Combined ISH for LFNG and Hoechst nuclear staining. Bar $=50 \mu \mathrm{m}$.

Additional file 9: Fig. S7. Molecular heterogeneity within the RP $(A, D, G)$ Expression of three genes in the central domain of the RP (delimited by dotted lines). $(\mathrm{B}, \mathrm{E}, \mathrm{H}) \mathrm{Rspol}$ is preferentially expressed in the RP periphery. $(C, F, I)$ Combination of adjacent sections showing complementary expression of the above. (J) Schematic representation of the expression pattern of several genes upregulated at the RP stage to either its medial $(M, r e d)$, lateral $(L$, green), to both domains (green+red) or to NT regions except for the RP (blue). Bar $=50 \mu \mathrm{m}$.

Additional file 10: Fig. S8. Expression patterns of Notch ligands and receptors. $(A, D, G, J, M)$ Expression of three Notch ligands $(A, D, G)$ and the Notch1 and 2 receptors $(J, M)$ at the RP stage. $(\mathrm{B}, \mathrm{E}, \mathrm{H}, \mathrm{K}, \mathrm{N})$ Rspol expression in adjacent sections. $(C, F, I, L, O)$ Overlay of the above. Note that the dorsal limit of DII1, Jagged1 and Jagged2 mRNA expression corresponds to the ventral limit of the RP. Notch1 and Notch2 mRNAs are expressed apically in the RP domain. Bar= 50um.

Additional file 11: Fig. S9. Missexpression of active Notch1 (CNIC) in quail neural tubes prevents the upregulation of RP markers. Left column represents electroporation of CNIC-GFP (A,D,G). Middle column depicts ISH for BAMBI (N=3), Raldh2 $(N=5)$ or Draxin $(N=4)$, and right column is an overlay of the precedent, respectively. Note that CNIC-treated cells are devoid or have reduced marker expression (arrows). For control GFP see Fig. 4. Bar $=50 \mu \mathrm{m}$.

Additional file 12: Fig. S10. Neural crest formation is preserved in the absence of Notch signaling. (A-F) Transverse sections through the dorsal neural tube at the level of the forelimb in wildtype (WT) and Wnt1-Cre; $\mathrm{Mib}^{\mathrm{ffffl}}$ mouse embryos, immunostained for AP2a labeling neural crest and dorsal root ganglion (DRG) neurons (green), and cleaved-caspase 3 labeling apoptotic cells (red). At all stages examined, no cell death is evident in the premigratory neural crest or presumptive roof plate region. At E11.5 (E, F) increased cell death is evident in the DRG of Wnt1-Cre; Mib $1^{f / f f l}$ embryos as previously reported (arrows). Bar $=100 \mu \mathrm{m} .(\mathrm{G}, \mathrm{H})$ Transverse sections at E10.5 immunostained for DLL1 (green) and EdU (red) following a $1 \mathrm{hr}$ EdU pulse to label proliferating cells. The dashed line indicates the boundary of DLL1 expression and defines the region used for quantification in (I). (I) Quantification of the number of EdUpositive proliferating cells in the DII1-negative domain (presumptive roof plate) at E10.5, expressed as a percentage of the total number of DAPIpositive nuclei. Note the reduction in proliferating cells present in Wnt1Cre; Mib ${ }^{f / f l}$ embryos compared to wildtype. $N=6$ embryos, ${ }^{* *} p=0.0018$. Bar $=50 \mu \mathrm{m}$.

Additional file 13: Table S3. List of primers used for both quail and mouse in situ hybridization.

\section{Acknowledgements}

We thank llana Blech for technical assistance with the avian part of the study; Tamar Hashimshony, Tal Katz-Ezov, and Olga Karinsky from the Genome Center at the Technion, for assistance with the RNA-seq. We are indebted to Sharona Elgavish, Yuval Nevo, and Hadar Benyamini for help with bioinformatic analysis, and Avihu Klar for helpful suggestions. We also thank Xiangjun Xu for technical assistance with mouse genotyping.

\section{Authors' contributions}

CK and QS conceived the project and all authors designed the experiments; SO performed the transcriptome analysis and all experiments in quail embryos; SW performed the mouse embryo experiments; SK and MT assisted with probe preparation and ISH analysis; and CK wrote the manuscript. All authors discussed and agreed on the results and approved the manuscript.

\section{Funding}

This study was supported by grants from the Israel Science Foundation (ISF \#209/18) to CK and the NHMRC (APP1144004) to SW and QS.

Availability of data and materials

Raw RNAseq files and materials will be provided upon request.

\section{Declarations}

Ethics approval and consent to participate

Not applicable.

\section{Consent for publication}

Not applicable.

\section{Competing interests}

No competing interests declared.

Received: 21 December 2020 Accepted: 25 March 2021

Published online: 23 April 2021

\section{References}

1. Krispin S, Nitzan E, Kalcheim C. The dorsal neural tube: a dynamic setting for cell fate decisions. Dev Neurobiol. 2010;70(12):796-812. https://doi.org/10.1 002/dneu.20826.

2. Le Douarin NM, Kalcheim C. The neural crest. 2nd ed. New York: Cambridge University Press; 1999. https://doi.org/10.1017/CBO9780511897948.

3. Kalcheim C, Kumar D. Cell fate decisions during neural crest ontogeny. Int J Dev Biol. 2017;61(3-4-5):195-203. https://doi.org/10.1387/ijdb.160196ck.

4. Martik ML, Bronner ME. Regulatory logic underlying diversification of the neural crest. Trends Genet. 2017;33(10):715-27. https://doi.org/10.1016/j.tig.2 017.07.015.

5. Nitzan E, Avraham O, Kahane N, Ofek S, Kumar D, Kalcheim C. Dynamics of BMP and Hes1/Hairy1 signaling in the dorsal neural tube underlies the transition from neural crest to definitive roof plate. BMC Biol. 2016;14(1):23. https://doi.org/10.1186/s12915-016-0245-6.

6. Andrews MG, Kong J, Novitch BG, Butler SJ. New perspectives on the mechanisms establishing the dorsal-ventral axis of the spinal cord. Curr Top Dev Biol. 2019;132:417-50. https://doi.org/10.1016/bs.ctdb.2018.12.010

7. Chizhikov W, Millen KJ. Mechanisms of roof plate formation in the vertebrate CNS. Nat Rev Neurosci. 2004;5(10):808-12. http://www.ncbi.nlm. nih.gov/entrez/query.fcgi?cmd=Retrieve\&db=PubMed\&dopt=Citation\&list uids=15378040. https://doi.org/10.1038/nrn1520. 
8. Le Dreau G, Marti E. Dorsal-ventral patterning of the neural tube: a tale of three signals. Dev Neurobiol. 2012;72(12):1471-81. https://doi.org/10.1002/ dneu.22015.

9. le Dréau G, Garcia-Campmany L, Angeles Rabadán M, Ferronha T, Tozer S, Briscoe J, et al. Canonical BMP7 activity is required for the generation of discrete neuronal populations in the dorsal spinal cord. Development. 2012; 139(2):259-68. https://doi.org/10.1242/dev.074948.

10. Lee KJ, Mendelsohn M, Jessell TM. Neuronal patterning by BMPs: a requirement for GDF7 in the generation of a discrete class of commissural interneurons in the mouse spinal cord. Genes Dev. 1998;12(21):3394-407. https://doi.org/10.1101/gad.12.21.3394.

11. Duval N, Vaslin C, Barata TC, Frarma Y, Contremoulins V, Baudin X, et al. BMP4 patterns Smad activity and generates stereotyped cell fate organization in spinal organoids. Development. 2019;146:doi: https://doi. org/10.1242/dev.175430

12. Timmer JR, Wang C, Niswander L. BMP signaling patterns the dorsal and intermediate neural tube via regulation of homeobox and helix-loop-helix transcription factors. Development. 2002;129(10):2459-72. http://www.ncbi. nlm.nih.gov/entrez/query.fcgi? $c$ nd=Retrieve\&db=PubMed\&dopt=Cita tion\&list_uids=11973277.

13. Andrews MG, Del Castillo LM, Ochoa-Bolton E, Yamauchi K, Smogorzewski J Butler SJ. BMPs direct sensory interneuron identity in the developing spinal cord using signal-specific not morphogenic activities. Elife. 2017;6:doi: https://doi.org/10.7554/eLife.30647

14. Zechner D, Muller T, Wende H, Walther I, Taketo MM, Crenshaw EB 3rd, et al. Bmp and Wnt/beta-catenin signals control expression of the transcription factor Olig3 and the specification of spinal cord neurons. Dev Biol. 2007:303(1):181-90. https://doi.org/10.1016/j.ydbio.2006.10.045.

15. Shinozuka T, Takada R, Yoshida S, Yonemura S, Takada S. Wnt produced by stretched roof-plate cells is required for the promotion of cell proliferation around the central canal of the spinal cord. Development. 2019;146(2): dev159343. https://doi.org/10.1242/dev.159343.

16. Xing L, Anbarchian T, Tsai JM, Plant GW, Nusse R. Wnt/beta-catenin signaling regulates ependymal cell development and adult homeostasis. Proc Natl Acad Sci U S A. 2018;115(26):E5954-62. https://doi.org/10.1073/ pnas.1803297115.

17. Hunter NL, Dymecki SM. Molecularly and temporally separable lineages form the hindbrain roof plate and contribute differentially to the choroid plexus. Development. 2007;134(19):3449-60. https://doi.org/10.1242/dev.003 095.

18. Broom ER, Gilthorpe JD, Butts T, Campo-Paysaa F, Wingate RJT. The roof plate boundary is a bi-directional organiser of dorsal neural tube and choroid plexus development. Dev. 2012;139(22):4261-70. https://doi.org/1 0.1242/dev.082255.

19. Altman J, Bayer SA. The development of the rat spinal cord. Adv Anat Embryol Cell Biol. 1984;85:1-164. http://www.ncbi.nlm.nih.gov/pubmed/ 6741688. https://doi.org/10.1007/978-3-642-69537-7_1.

20. Bohme G. Formation of the central canal and dorsal glial septum in the spinal cord of the domestic cat. J Anat. 1988;159:37-47. http//uww.ncbi.nlm.nih.gov/pubmed/3248971

21. Canizares MA, Albors AR, Singer G, Suttie N, Gorkic M, Felts P, et al. Multiple steps characterise ventricular layer attrition to form the ependymal cell lining of the adult mouse spinal cord central canal. J Anat. 2019;236(2):33450. https://doi.org/10.1111/joa.13094.

22. Kridsada K, Niu J, Haldipur P, Wang Z, Ding L, Li JJ, Lindgren AG, Herrera E, Thomas GM, Chizhikov W, Millen KJ, Luo W. Roof plate-derived radial gliallike cells support developmental growth of rapidly adapting mechanoreceptor ascending axons. Cell Rep. 2018;23(10):2928-41. https:// doi.org/10.1016/j.celrep.2018.05.025.

23. Ghazale H, Ripoll C, Leventoux N, Jacob L, Azar S, Mamaeva D, Glasson Y, Calvo CF, Thomas JL, Meneceur S, Lallemand Y, Rigau V, Perrin FE, Noristani HN, Rocamonde B, Huillard E, Bauchet L, Hugnot JP. RNA profiling of the human and mouse spinal cord stem cell niches reveals an embryonic-like regionalization with MSX1(+) roof-plate-derived cells. Stem Cell Reports. 2019:12(5):1159-77. https://doi.org/10.1016/j.stemcr.2019.04.001.

24. Kahane N, Kalcheim C. Identification of early postmitotic cells in distinct embryonic sites and their possible roles in morphogenesis. Cell Tissue Res. 1998;294(2):297-307. https://doi.org/10.1007/s004410051180.

25. Krispin S, Nitzan E, Kassem Y, Kalcheim C. Evidence for a dynamic spatiotemporal fate map and early fate restrictions of premigratory avian neural crest. Development. 2010;137(4):585-95. https://doi.org/10.1242/dev. 041509
26. Nitzan E, Krispin S, Pfaltzgraff ER, Klar A, Labosky P, Kalcheim C. A dynamic code of dorsal neural tube genes regulates the segregation between neurogenic and melanogenic neural crest cells. Development. 2013;140(11): 2269-79. https://doi.org/10.1242/dev.093294.

27. Kamata T, Katsube K, Michikawa M, Yamada M, Takada S, Mizusawa H. Rspondin, a novel gene with thrombospondin type 1 domain, was expressed in the dorsal neural tube and affected in Wnts mutants. Biochim Biophys Acta. 2004;1676:51-62. http://www.ncbi.nlm.nih.gov/pubmed/14732490

28. Artavanis Tsakonas S, Rand MD, Lake RJ. Notch signaling: cell fate control and signal integration in development. Science. 1999;284:770-6.

29. Lai EC. Notch signaling: control of cell communication and cell fate. Development. 2004;131(5):965-73. http://www.ncbi.nlm.nih.gov/entrez/ query.fcgi?cmd=Retrieve\&db=PubMed\&dopt=Citation\&list_uids $=14973298$. https://doi.org/10.1242/dev.01074.

30. Henrique D, Schweisguth F. Mechanisms of notch signaling: a simple logic deployed in time and space. Dev. 2019;146(3):dev172148. https://doi.org/1 $0.1242 /$ dev.172148.

31. Rolo A, Galea GL, Savery D, Greene NDE, Copp AJ. Novel mouse model of encephalocele: post-neurulation origin and relationship to open neural tube defects. Dis Model Mech. 2019;12:doi:https://doi.org/10.1242/dmm.040683.

32. Chizhikov W, Millen KJ. Roof plate-dependent patterning of the vertebrate dorsal central nervous system. Dev Biol. 2005;277(2):287-95. http://www. ncbi.nlm.nih.gov/entrez/query.fcgi?cmd=Retrieve\&db=PubMed\&dopt=Cita tion\&list_uids=15617675. https://doi.org/10.1016/j.ydbio.2004.10.011.

33. Muroyama $Y$, Fujihara M, Ikeya M, Kondoh H, Takada S. Wnt signaling plays an essential role in neuronal specification of the dorsal spinal cord. Genes Dev. 2002;16(5):548-53. https://doi.org/10.1101/gad.937102.

34. Knoepfler PS, Cheng PF, Eisenman RN. N-myc is essential during neurogenesis for the rapid expansion of progenitor cell populations and the inhibition of neuronal differentiation. Genes Dev. 2002;16(20):2699-712. https://doi.org/10.1101/gad.1021202.

35. Nitzan E, Krispin S, Pfaltzgraff ER, Klar A, Labosky PA, Kalcheim C. A dynamic code of dorsal neural tube genes regulates the segregation between neurogenic and melanogenic neural crest cells. Development. 2012;140:2269-79.

36. Shoval I, Ludwig A, Kalcheim C. Antagonistic roles of full-length $\mathrm{N}$-cadherin and its soluble BMP cleavage product in neural crest delamination. Development. 2007;134:491-501.

37. Wiszniak S, Schwarz Q. Notch signalling defines dorsal root ganglia neuroglial fate choice during early neural crest cell migration. BMC Neurosci. 2019:20(1):1-13. https://doi.org/10.1186/s12868-019-0501-0.

38. Kang K, Lee D, Hong S, Park SG, Song MR. The E3 ligase mind bomb-1 (Mib1) modulates delta-notch signaling to control neurogenesis and gliogenesis in the developing spinal cord. J Biol Chem. 2013;288(4):2580-92. https://doi.org/10.1074/jbc.M112.398263.

39. Sela-Donenfeld D, Kalcheim C. Regulation of the onset of neural crest migration by coordinated activity of BMP4 and Noggin in the dorsal neural tube. Development. 1999;126(21):4749-62.

40. Delile J, Rayon T, Melchionda M, Edwards A, Briscoe J, Sagner A. Single cell transcriptomics reveals spatial and temporal dynamics of gene expression in the developing mouse spinal cord. Development. 2019;146 doi:https://doi. org/10.1242/dev.173807:1-14.

41. Millen KJ, Millonig JH, Hatten ME. Roof plate and dorsal spinal cord dl1 interneuron development in the dreher mutant mouse. Dev Biol. 2004; 270(2):382-92. http://www.ncbi.nlm.nih.gov/entrez/query.fcgi?cmd= Retrieve\&db=PubMed\&dopt=Citation\&list_uids=15183721. https://doi.org/1 0.1016/j.ydbio.2004.03.008

42. Lee KJ, Dietrich $P$, Jessell TM. Genetic ablation reveals that the roof plate is essential for dorsal interneuron specification. Nature. 2000;403(6771):734-40. https://doi.org/10.1038/35001507.

43. Bronner ME. Formation and migration of neural crest cells in the vertebrate embryo. Histochem Cell Biol. 2012;138(2):179-86. https://doi.org/10.1007/ s00418-012-0999-z.

44. Burstyn-Cohen T, Stanleigh J, Sela-Donenfeld D, Kalcheim C. Canonical Wnt activity regulates trunk neural crest delamination linking BMP/noggin signaling with G1/S transition. Development. 2004;131(21):5327-39. https:// doi.org/10.1242/dev.01424.

45. Higashihori N, Song Y, Richman JM. Expression and regulation of the decoy bone morphogenetic protein receptor BAMBI in the developing avian face. Dev Dyn. 2008;237(5):1500-8. https://doi.org/10.1002/dvdy.21529.

46. Maden $M$, Ong DE, Summerbell D, Chytil F, Hirst EA. Cellular retinoic acidbinding protein and the role of retinoic acid in the development of the 
chick embryo. Dev Biol. 1989;135(1):124-32. https://doi.org/10.1016/0012-1 606(89)90163-2.

47. Qin Z, Ren F, Xu X, Ren Y, Li H, Wang Y, Zhai Y, Chang Z. ZNF536, a novel zinc finger protein specifically expressed in the brain, negatively regulates neuron differentiation by repressing retinoic acid-induced gene transcription. Mol Cell Biol. 2009;29(13):3633-43. https://doi.org/10.1128/ MCB.00362-09.

48. de Lau W, Peng WC, Gros P, Clevers H. The R-spondin/Lgr5/Rnf43 module: regulator of Wnt signal strength. Genes Dev. 2014;28(4):305-16. https://doi org/10.1101/gad.235473.113.

49. Binnerts ME, Kim KA, Bright JM, Patel SM, Tran K, Zhou M, Leung JM, Liu Y, Lomas WE, Dixon M, Hazell SA, Wagle M, Nie WS, Tomasevic N, Williams J, Zhan X, Levy MD, Funk WD, Abo A. R-Spondin1 regulates Wnt signaling by inhibiting internalization of LRP6. Proc Natl Acad Sci U S A. 2007;104(37): 14700-5. https://doi.org/10.1073/pnas.0702305104.

50. Kim KA, Wagle M, Tran K, Zhan X, Dixon MA, Liu S, Gros D, Korver W, Yonkovich S, Tomasevic N, Binnerts M, Abo A. R-Spondin family members regulate the Wnt pathway by a common mechanism. Mol Biol Cell. 2008; 19(6):2588-96. https://doi.org/10.1091/mbc.e08-02-0187.

51. Deng C, Reddy P, Cheng Y, Luo CW, Hsiao CL, Hsueh AJW. Multi-functional norrin is a ligand for the LGR4 receptor. J Cell Sci. 2013;126(9):2060-8. https://doi.org/10.1242/jcs.123471.

52. Liu Y, Helms AW, Johnson JE. Distinct activities of MsX1 and MsX3 in dorsal neural tube development. Development. 2004;131(5):1017-28. https://doi. org/10.1242/dev.00994.

53. Cheng YC, Amoyel M, Qiu X, Jiang YJ, Xu Q, Wilkinson DG. Notch activation regulates the segregation and differentiation of rhombomere boundary cells in the zebrafish hindbrain. Dev Cell. 2004;6(4):539-50. https://doi.org/1 0.1016/S1534-5807(04)00097-8.

54. Baek JH, Hatakeyama J, Sakamoto S, Ohtsuka T, Kageyama R. Persistent and high levels of Hes1 expression regulate boundary formation in the developing central nervous system. Development. 2006;133:2467-76. https://doi.org/10.1242/dev.02403.

55. Zeltser LM, Larsen CW, Lumsden A. A new developmental compartment in the forebrain regulated by lunatic fringe. Nat Neurosci. 2001;4(7):683-4. https://doi.org/10.1038/89455.

56. Endo Y, Osumi N, Wakamatsu Y. Bimodal functions of Notch-mediated signaling are involved in neural crest formation during avian ectoderm development. Development. 2002;129(4):863-73.

57. Duval N, Daubas P, Bourcier de Carbon C, St Cloment C, Tinevez JY, Lopes $M$, et al. Msx1 and Msx2 act as essential activators of Atoh1 expression in the murine spinal cord. Development. 2014;141(8):1726-36. https://doi.org/1 $0.1242 /$ dev.099002.

58. Wine-Lee L, Ahn K, Richardson RD, Mishina Y, Lyons KM, Crenshaw EB. Signaling through BMP type 1 receptors is required for development of interneuron cell types in the dorsal spinal cord. Development. 2004;131(21): 5393-403. https://doi.org/10.1242/dev.01379.

59. Monsoro-Burq AH, Duprez D, Watanabe Y, Bontoux M, Vincent C, Brickell P, le Douarin $\mathrm{N}$. The role of bone morphogenetic proteins in vertebral development. Development. 1996;122(11):3607-16.

60. Wakamatsu Y, Maynard TM, Weston JA. Fate determination of neural crest cells by NOTCH-mediated lateral inhibition and asymmetrical cell division during gangliogenesis. Development. 2000;127(13):2811-21.

61. Koo BK, Lim HS, Song R, Yoon MJ, Yoon KJ, Moon JS, Kim YW, Kwon MC, Yoo KW, Kong MP, Lee J, Chitnis AB, Kim CH, Kong YY. Mind bomb 1 is essential for generating functional notch ligands to activate notch. Development. 2005;132(15):3459-70. https://doi.org/10.1242/dev.01922.

62. Shimizu K, Chiba S, Saito T, Takahashi T, Kumano K, Hamada Y, Hirai H. Integrity of intracellular domain of Notch ligand is indispensable for cleavage required for release of the Notch2 intracellular domain. EMBO J. 2002;21 (3):294-302. http://www.ncbi.nlm.nih.gov/entrez/query.fcgi?cmd= Retrieve\&db=PubMed\&dopt=Citation\&list_uids=11823422. https://doi.org/1 0.1093/emboj/21.3.294

63. Sato Y, Kasai T, Nakagawa S, Tanabe K, Watanabe T, Kawakami K, et al. Stable integration and conditional expression of electroporated transgenes in chicken embryos. Dev Biol. 2007;305:616-24. https://doi.org/10.1016/j. ydbio.2007.01.043.

64. Applebaum M, Ben-Yair R, Kalcheim C. Segregation of striated and smooth muscle lineages by a Notch-dependent regulatory network. BMC Biol. 2014; 12:doi:https://doi.org/10.1186/s12915-014-0053-9.
65. Wakamatsu Y, Maynard TM, Jones SU, Weston JA. NUMB localizes in the basal cortex of mitotic avian neuroepithelial cells and modulates neuronal differentiation by binding to NOTCH-1. Neuron. 1999;23(1):71-81. https:// doi.org/10.1016/S0896-6273(00)80754-0.

66. Ben-Yair R, Kalcheim C. Notch and bone morphogenetic protein differentially act on dermomyotome cells to generate endothelium, smooth, and striated muscle. J Cell Biol. 2008;180(3):607-18. https://doi.org/10.1083/ jcb.200707206.

67. Hashimshony T, Senderovich N, Avital G, Klochendler A, de Leeuw Y, Anavy L, Gennert D, Li S, Livak KJ, Rozenblatt-Rosen O, Dor Y, Regev A, Yanai I. CEL-Seq2: sensitive highly-multiplexed single-cell RNA-Seq. Genome Biol. 2016;17(1):77. https://doi.org/10.1186/s13059-016-0938-8.

68. Dottori M, Gross MK, Labosky P, Goulding M. The winged-helix transcription factor Foxd3 suppresses interneuron differentiation and promotes neural crest cell fate. Development. 2001;128(21):4127-38.

69. Kos R, Reedy MV, Johnson RL, Erickson CA. The winged-helix transcription factor FoxD3 is important for establishing the neural crest lineage and repressing melanogenesis in avian embryos. Development. 2001;128(8): 1467-79.

70. Nieto MA, Sargent MG, Wilkinson DG, Cooke J. Control of cell behavior during vertebrate development by slug, a zinc finger gene. Science. 1994; 264:835-9.

71. Schubert FR, Sobreira DR, Janousek RG, Alvares LE, Dietrich S. Dact genes are chordate specific regulators at the intersection of Wnt and Tgf- $\beta$ signaling pathways. BMC Evol Biol. 2014;14:doi: https://doi.org/10.1186/14 71-2148-14-157.

72. Sienknecht UJ, Fekete DM. Mapping of Wnt, frizzled, and Wnt inhibitor gene expression domains in the avian otic primordium. J Comp Neurol. 2009; 517(6):751-64. https://doi.org/10.1002/cne.22169.

73. Schwarz Q, Gu C, Fujisawa H, Sabelko K, Gertsenstein M, Nagy A, et al. Vascular endothelial growth factor controls neuronal migration and cooperates with Sema3A to pattern distinct compartments of the facial nerve. Genes Dev. 2004;18:2822-34. https://doi.org/10.1101/gad.322904.

74. Cau E, Gradwohl G, Fode C, Guillemot F. Mash1 activates a cascade of bHLH regulators in olfactory neuron progenitors. Development. 1997;124(8):161121

\section{Publisher's Note}

Springer Nature remains neutral with regard to jurisdictional claims in published maps and institutional affiliations.
Ready to submit your research? Choose BMC and benefit from:

- fast, convenient online submission

- thorough peer review by experienced researchers in your field

- rapid publication on acceptance

- support for research data, including large and complex data types

- gold Open Access which fosters wider collaboration and increased citations

- maximum visibility for your research: over $100 \mathrm{M}$ website views per year

At BMC, research is always in progress.

Learn more biomedcentral.com/submissions 